\title{
MOTIVES ASSOCIATED TO GRAPHS
}

\author{
SPENCER BLOCH
}

AbstraCt. A report on recent results and outstanding problems concerning motives associated to graphs.

\section{INTRODUCTION}

The theory of Feynman diagrams as a way to write series expansions for perturbations of Gaussian integrals is by now old enough to be classical. (Classical in mathematics can be defined to be that which has fallen off the active shelves of your neighborhood mathematical library and is stored somewhere "below stairs". It varies as a function of the library.) And yet, somehow, the subject remains an active one. Why is this? Physicists, as Dirk Kreimer never tires of telling me, actually need in their experiments the numbers which the theory produces. (As far as I can tell, a particle in physics is basically a particularly sweet array of Feynman diagrams.) For mathematicians, it has become clear in the finite dimensional case that rewriting a Gaussian type integral as a sum over graphs is a profound reworking of the basic mathematical object which can yield deep and surprising insights.

The infinite dimensional case, which is the inspiration for this paper, is mathematically more mysterious. As an algebraic geometer interested in motives, the author was attracted by the results of Broadhurst and Kreimer [BK1], [BK2] who showed that in numerous cases, the Feynman amplitudes which are periods in the motivic sense and which give the coefficients of the perturbative series, are multiple zeta numbers. The Feynman procedure yields integrals over $\mathbb{P}^{n}(\mathbb{R})$ with an algebraic integrand whose denominator is a product of quadrics. Using a trick of Schwinger, this integral is rewritten as an integral over a simplex where the integrand involves the classical graph polynomial associated to the particular graph. It is clear from op. cit. that the central case of interest is the case of a logarithmically divergent graph

Date: Oct. 20, 2006. 
$\Gamma$, where the number $2 n$ of edges is twice the loop number: $n=h_{1}(\Gamma)$. In this case the Schwinger integral is

$$
\int_{\sigma} \frac{\Omega}{\Psi^{2}}
$$

where $\sigma$ is the simplex in projective space $\mathbb{P}^{2 n-1}, \Omega=\sum_{1}^{2 n}(-1)^{i} A_{i} d A_{1} \wedge$ $\ldots \widehat{d A_{i}} \ldots d A_{2 n}$ is the top degree form of homogeneous degree the number of edges and $\Psi$ is the graph polynomial, which is homogeneous of degree equal to the loop number.

So, roughly speaking, the geometry we need to understand is the geometry of a pair $\left(\mathbb{P}^{2 n-1}-X, \Delta\right)$, where $X: \Psi=0$ is the graph hypersurface, and $\Delta: \prod_{i=1}^{2 n} A_{i}=0$ is the coordinate simplex. Here $\sigma=\left\{\left(a_{1}, \ldots, a_{2 n}\right) \mid a_{i} \geq 0\right\}$, so the boundary of the chain $\sigma$ is supported on $\Delta$. The combinatorics of the intersection $\sigma \cap X$ is the main focus of our effort. The basic geometry is described in Section 6. In Sections 7 and 8 we describe the differential Hopf algebra in the category of motives which encodes this geometry. The key idea is Proposition 6.3 which describes the normal cone to a coordinate linear space contained in the graph hypersurface $X$. By way of example, in Section 9 we give some details for the example of the wheel with three spokes. Finally, in Section 10 we discuss the problem of renormalization. From our geometric viewpoint, renormalization is necessary when the integrand in (1.1) acquires poles along some exceptional divisors when we blow up faces of $\Delta$.

The reader will discover that, in fact, there is precious little in these notes about multiple zeta numbers and Feynman amplitudes. Instead, the focus is on the Hopf algebra properties of the motivic construction. In truth, the relationship between graphs and multiple zeta numbers is still completely unclear.

This is intended to be an expository account, so I have tried to structure things so the material grows increasingly technical toward the end of the paper. Sections 2 and 3 are sort of standard attempts to go back to the roots and see where the graphs come in. Section 4 exposes the linear algebra of the graph polynomial. Of interest here is the unified treatment of the graph polynomial and the canonical quadratic form $R$ (cf. Proposition 4.4) on the space of external momenta. The idea is that the ultimate period formula (5.5) which involves both the graph polynomial and external momenta should be seen as a natural consequence of the basic linear algebra. (By the way, I learned formula (5.5) from the book [IZ]. Although perhaps a bit "long in the tooth" now for physicists, this book is excellent.) 
This paper is based on my work with H. Esnault and D. Kreimer, [BEK]. That they do not appear as co-authors is based on constraints on collaboration imposed by time and distance (well understood concepts in physics). I would also like to acknowledge helpful correspondence with K. Yeats.

What follows is not a survey. I lack the depth of knowledge to do a good survey. Were I to try, I would include some of the original calculations in [BK1], [BK2], the work of Connes-Kreimer [CK1], [CK2] as well as the more recent work of Connes-Marcolli [CM]. Certainly the work of Brosnan-Belkale [BB] showing that the full motive of graph hypersurfaces is not in general mixed Tate should be discussed. Finally, I would also include an account of the paper of Goncharov-Manin on multiple zeta numbers arising from motives on the moduli space of stable $n$-pointed curves of genus $0[\mathrm{GM}]$, as well as the recent thesis of F. Brown on the same subject [Br]. Unlike the case of graphs where the picture is still mysterious, the genus 0 moduli story is quite complete.

I would like to thank the referee for a very careful report, pointing out, in particular, the need for $|v|$ ! in the statement of Theorem 2.10.

Finally an apology for my totally imprecise use of the word "motive". Because of technical problems associated with defining motives for cohomology groups of open and singular varieties, it would really be better to work systematically with Hodge structures. Indeed, in sections 9 and 10 I do this. However, the spirit of this program is motivic. Multiple zeta numbers are motivic. The name of the game is to construct geometric objects associated to graphs, not just Hodge structures. The theory of motives was intended as a tool to study concrete geometric and arithmetic objects, so I have kept the term.

\section{Perturbed Gaussian Integration}

The origin of our subject, the moment, so to speak, the physical Dorothy enters the mathematical land of $\mathrm{Oz}$, is possible to identify with some precision; and it seems worthwhile to say a few words about this. I follow $[\mathrm{P}]$ and $[\mathrm{IZ}]$. The Feynman graph method in finite dimensions grows from an attempt to calculate an integral of the form

$$
Z_{U}:=\int_{\mathbb{R}^{d}} \exp \left(\frac{-1}{2}\langle A x, x\rangle+h U(x)\right) d x .
$$

Here $A$ is a positive-definite symmetric $d \times d$ matrix over $\mathbb{R}, h$ is a parameter, and we do not worry too much about convergence. We write $Z_{0}$ for the Gaussian integral, $U=0$.

As inputs to our calculation, we use two formal algebraic results. Let $f_{1}, \ldots, f_{m}$ be polynomial functions on $\mathbb{R}^{d}$. The correlation function is 
defined by

$$
\left\langle f_{1}, \ldots, f_{m}\right\rangle:=Z_{0}^{-1} \int_{\mathbb{R}^{d}} \exp \left(\frac{-1}{2}\langle A x, x\rangle\right) f_{1}(x) \cdots f_{m}(x) d x .
$$

A standard Gaussian integral calculation yields

$$
\left\langle f_{1}, \ldots, f_{m}\right\rangle=\left.f_{1}\left(\frac{\partial}{\partial b}\right) \cdots f_{m}\left(\frac{\partial}{\partial b}\right) \exp \left(\frac{1}{2}\left\langle b, A^{-1} b\right\rangle\right)\right|_{b=0} .
$$

The second formal calculation, Wick's theorem, says

$$
\left.\partial_{i_{1}} \cdots \partial_{i_{m}} \exp \left(\frac{1}{2}\left\langle b, A^{-1} b\right\rangle\right)\right|_{b=0}= \begin{cases}0 & m \text { odd } \\ \sum A^{i_{j_{1}} i_{j_{2}}} \cdots A^{i_{j_{m-1}} i_{j_{m}}} & m=2 r \text { even }\end{cases}
$$

Here $A^{-1}=\left(A^{i j}\right)$, and the above sum is over all partitions into subsets with two elements

$$
\left\{i_{1}, \ldots, i_{m}\right\}=\left\{i_{j_{1}}, i_{j_{2}}\right\} \amalg \ldots \amalg\left\{i_{j_{m-1}}, i_{j_{m}}\right\} .
$$

(Confusing point: It is important to distinguish between decompositions and partitions of a set. A partition should be thought of as an equivalence relation. $\{12\}\{34\}$ and $\{43\}\{12\}$ are the same partition of $\{1234\}$ into two subsets with 2 elements but different decompositions. For $m=2 r$, each partition corresponds to $r ! 2^{r}$ decompositions. Wick's theorem can be restated by inserting $\frac{1}{r ! 2^{r}}$ on the right and summing over decompositions.)

(Another confusing point: even if various of the $i_{j}$ coincide, there are always $1 \cdot 3 \cdots(2 r-1)$ such partitions.)

Formally, at least, our integral can be written

$$
Z_{U} / Z_{0}=\left.e^{h U\left(\frac{\partial}{\partial b}\right)} e^{\frac{1}{2}\left\langle b, A^{-1} b\right\rangle}\right|_{b=0} .
$$

Suppose for simplicity that $U=\sum_{I} U_{I} x^{I}$ is a homogeneous polynomial of degree $p>2$. Here if $I=\left\{i_{1}, \ldots, i_{p}\right\}$ then $x^{I}:=x_{i_{1}} \ldots x_{i_{p}}$. Also $U_{I}$ is symmetric with respect to permutations of $I$. If we isolate the terms of degree $n$ in $h$, we are led to apply Wick's theorem to expressions like

$$
\begin{gathered}
\left.\frac{1}{n !} \sum_{I_{1}, \ldots, I_{n}} U_{I_{1}} \cdots U_{I_{n}} \frac{\partial^{I_{1}+\ldots+I_{n}}}{\partial b_{I_{1}+\ldots+I_{n}}} e^{\frac{1}{2}\left\langle b, A^{-1} b\right\rangle}\right|_{b=0}= \\
\frac{1}{n !} \sum_{I} U_{I_{1}} \cdots U_{I_{n}} \sum_{I=\left\{j_{1}, j_{2}\right\} \amalg \ldots \amalg\left\{j_{n p-1}, j_{n p}\right\}} A^{j_{1} j_{2}} \cdots A^{j_{n p-1} j_{n p}} .
\end{gathered}
$$

( $I:=I_{1} \amalg \ldots \amalg I_{n}$, with $n p$ assumed even. Again as remarked above, partitions are defined formally on the set $I$ without regard to the possibility that elements of $I$ coincide.) 
This is where graphs appear, and it is important to be precise, because confusion is possible. (As mathematicians, clarity and precision are part of our "mission civilisatrice".) A graph is determined by a finite set $H E$, (the set of $1 / 2$-edges) together with two equivalence conditions, with quotients denoted $V$ (vertices) and $E$ (edges).

$$
V \leftarrow H E \rightarrow E
$$

We further suppose the equivalence classes in $E$ each have 2 elements. An automorphism of a graph is a $\sigma \in A u t(H E)$ such that there exist isomorphisms $\sigma_{E}: E \cong E, \sigma_{V}: V \cong V$ making the diagram

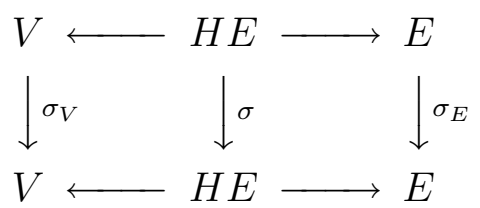

commute.

We write $|\operatorname{Aut}(\Gamma)|$ for the order of the group of automorphisms of the graph $\Gamma$ and $|\Gamma|$ for the number of vertices.

We will consider graphs labeled by the set $\{1, \ldots, d\}$, which simply means we are given a mapping

$$
\ell: H E \rightarrow\{1, \ldots, d\}
$$

The basic result is

Theorem 2.1. We have the formal expansion

$$
Z_{U} / Z_{0}=\sum_{\Gamma} \frac{h^{|\Gamma|}}{|A u t(\Gamma)|} \sum_{H E(\Gamma) \stackrel{\ell}{\rightarrow}\{1, \ldots, d\}} \prod_{v \text { vertex }}|v| ! U_{\ell(v)} \prod_{e \text { edge }} A^{\ell(e)}
$$

Here $\ell(v)$ and $\ell(e)$ are the evident sets of indices, and $|v|$ denotes the number of half-edges meeting $v$.

Remark 2.2. I am endebted to the referee for pointing out the need for the $|v|$ !. If $\Gamma$ is a graph with $n r$-valent vertices and $n r$ half-edges, then

$$
\#\{\text { graphs of type } \Gamma\} \# \operatorname{Aut}(\Gamma)=n !(r !)^{n} \text {. }
$$

This can be rewritten

$$
\#\{\text { graphs of type } \Gamma\} / n !=(r !)^{n} / \# \operatorname{Aut}(\Gamma)
$$

which explains the $r$ !. 


\section{Infinite Dimensions}

The effect of introducing graphs is to reduce an integral (2.1) to a sum (2.10). One approach to QFT starts with an infinite dimensional analogue of the perturbed gaussian integral (2.1). The perturbative expansion (2.10) now involves integration rather than summation, but the integrals in question are finite dimensional. In place of $\mathbb{R}^{d}$ one takes some infinite dimensional space of fields $\phi$. (Think of $\phi$ as lying in some space of smooth functions on $\mathbb{R}^{4}$ with rapid decay.) In place of the symmetric matrix $A$, one typically takes the Klein-Gordon differential operator

$$
K=-\partial^{2} / \partial x_{1}^{2}-\partial^{2} / \partial x_{2}^{2}-\partial^{2} / \partial x_{3}^{2}+\partial^{2} / \partial x_{0}^{2}+m^{2}
$$

In $\phi^{4}$-theory, the perturbation term is

$$
U(\phi):=\int_{\mathbb{R}^{4}} \phi^{4} d x_{1} \ldots d x_{4} .
$$

Finally, the set of labels $\{1, \ldots, d\}$ is replaced by the whole of $\mathbb{R}^{4}$. That is, $x \in \mathbb{R}^{4}$ defines a functional $\phi \mapsto \phi(x)$ in much the same way that $i \in\{1, \ldots, d\}$ defines a functional $\left(x_{1}, \ldots, x_{d}\right) \mapsto x_{i}$ on $\mathbb{R}^{d}$. The integral becomes

$$
Z_{U}=\int \mathcal{D} \phi \exp \left(\frac{-1}{2}\langle K \phi, \phi\rangle+h U(\phi)\right)
$$

(I've put the $-1 / 2$ to stress the parallel with (2.1). Typically in physics the coefficient is $\sqrt{-1} / 2$. Physicists refer to integrals like this as path integrals I suppose because in QED they are integrating over a space of paths. Whatever one calls it, it is more of a metaphor than an actual integral.)

The coordinates $A^{i j}$ for the inverse symmetric matrix in the finite dimensional case are replaced by the Green's function $G(x, y)$ associated to the operator $K$. This is a right inverse for $K$, which means that, as a distribution, it satisfies

$$
K_{x} G(x, y)=-\delta^{4}(x-y)
$$

Fourier theory yields

$$
G(x, y)=G(x-y)=\frac{1}{(2 \pi)^{4}} \int d^{4} p \frac{e^{-i p \cdot(x-y)}}{p^{2}+m^{2}}
$$

Here $x, y, k$ are 4 -vectors, and $p^{2}:=p \cdot p$. Note we have "Wick rotated", multiplying by $\sqrt{-1}$ to avoid the awkward indefinite quadratic form. We see from this that $G$ is translation-invariant. ( $p$ is momentum.) 
The finite dimensional perturbation term $\sum U_{I} x^{I}$ is replaced by

$$
\phi \mapsto \int_{\mathbb{R}^{4}} \phi(x)^{4} d x
$$

If we replace the sum in (2.10) by an integral, we are led to multiply integrals (3.5), one for each edge, and then integrate out the position variables. But from (3.6) there is one position variable for each vertex. If we (again formally) bring the position integration inside the momentum integrals (3.5) we end up first with an integral for each vertex of the form

$$
\int_{\mathbb{R}^{4}} \exp \left(x \cdot \sum \pm p_{j}\right) d x
$$

where the $p_{j}$ are the momentum variables corresponding to the edges meeting the given vertex, and the signs occur because of the $+x-y$. This integral gives the delta function for the hyperplane $\sum \pm p_{j}=0$. Next we have an outer integral over the product of all the integrands $\frac{1}{p_{j}^{2}+m^{2}}$. To simplify, we set the mass $m=0$. Our final integral has the $p_{j}^{2}$ in the denominator and has one $\delta$ function for each vertex.

Example 3.1. A simple but nontrivial example arises if we replace $\int \phi^{4} d x$ above with $\int \phi^{3} d x$. We may then take $\Gamma$ to be the graph given by the edges of a tetrahedron. It has 6 edges and 4 vertices. Suitably labeled, the 4 relations become

$$
p_{1}+p_{2}+p_{3}=0 ; p_{1}=p_{6}-p_{5} ; p_{2}=p_{4}-p_{6} ; p_{3}=p_{5}-p_{4}
$$

(Note there is one relation among the 4.) The integral becomes

$$
\int_{\mathbb{R}^{12}} \frac{d^{12} p}{p_{4}^{2} p_{5}^{2} p_{6}^{2}\left(p_{6}-p_{5}\right)^{2}\left(p_{6}-p_{4}\right)^{2}\left(p_{5}-p_{4}\right)^{2}}
$$

Sadly, this diverges. The physicist, however, looks at the corresponding projective integral over $\mathbb{P}^{11}$ which is viewed as a residue. The value is an elementary multiple of $\zeta(3)$.

To fix ideas, in the sequel when we talk about periods, we will usually have in mind a connected graph $\Gamma$ with $n$ loops and $2 n$ edges. Such a graph is said to be log divergent. Just as in the above example, this leads to a projective integral of the form

$$
\operatorname{Per}(q):=\int_{\mathbb{P}^{4 n-1}(\mathbb{R})} \frac{\Omega_{4 n-1}}{q_{1} \cdots q_{2 n}} ; \quad \Omega=\sum_{1}^{4 n}(-1)^{i-1} Z_{i} d Z_{1} \ldots \widehat{d Z}_{i} \ldots d Z_{4 n} .
$$


Here the $q_{i}$ are quadrics in $Z_{1}, \ldots, Z_{4 n}$. Indeed, if we look at the homology sequence

$$
0 \rightarrow H_{1}(\Gamma, \mathbb{Z}) \rightarrow \mathbb{Z}[E(\Gamma)] \rightarrow \mathbb{Z}[V(\Gamma)] \rightarrow H_{0}(\Gamma, \mathbb{Z}) \rightarrow 0
$$

where $E(\Gamma)$ and $V(\Gamma)$ are the edges and vertices, we see that $\Gamma$ has $n+1$ vertices. We start with $2 n$ copies of $\mathbb{R}^{4}$, one for each edge. We have $n+1 \delta$-functions, but there is one relation amongst them. Since each $\delta$-function drops the dimension by 4 , we end up with $\mathbb{R}^{4 n}$. The integrand is homogeneous, and we consider the projective integral.

We will discuss more general Feynman amplitudes in the context of external momenta in section 5 .

\section{Some Linear Algebra}

In this section, we assume our graph $\Gamma$ is connected, with $h_{1}(\Gamma)=n$ and $\# E(\Gamma)=m$. We truncate the exact sequence (3.11) of homology and define $W$ by the sequence

$$
0 \rightarrow H \rightarrow \mathbb{Z}[E(\Gamma)] \rightarrow W \rightarrow 0
$$

Definition 4.1. The momentum space associated to $\Gamma$ is the space $W$. In other words, the momentum space is the group of formal sums of vertices of degree 0 . (Of course, we may want to tensor with $\mathbb{R}$ or $\mathbb{C}$.)

The group $\mathbb{Z}[E(\Gamma)]$ is canonically self-dual simply by taking the dual basis to the basis given by edges $e \in E$. The functionals $e^{\vee}$ for $e \in E$ define linear forms by restriction on $H$. We view the squares of these functions $\left(e^{\vee}\right)^{2}: H \rightarrow \mathbb{Z}$ as rank 1 quadrics on $H$. If we choose a basis of $H$ we can associate a rank 1 symmetric matrix $N_{e}$ to $\left(e^{\vee}\right)^{2}$.

Definition 4.2. The Graph Polynomial

$$
\Psi_{\Gamma}:=\Psi_{H}=\operatorname{det}\left(\sum_{e \in E} A_{e} N_{e}\right)
$$

Note that this definition is independent of the choice of basis of $H$. Moreover, it would make sense for any subgroup $H \subset \mathbb{Z}[E]$, i.e. it depends only on the configuration defined by $H \subset \mathbb{Z}[E]$, not on the graph. For example, we may dualize and define a polynomial $\Psi_{W} \vee$ associated to $W^{\vee} \subset \mathbb{Z}[E]^{\vee} \cong \mathbb{Z}[E]$. It is easy to see (cf. [BEK], Prop. 1.6) that

$$
\Psi_{H}(A)=\left(\prod_{e \in E} A_{e}\right) \Psi_{W^{\vee}}\left(\ldots, A_{e}^{-1}, \ldots\right)
$$

$\Psi_{H}$ and $\Psi_{W} \vee$ are determinants of symmetric matrices $N_{H}$ and $N_{W}$ of sizes $n \times n$ and $(m-n) \times(m-n)$ respectively. Both matrices have 
entries linear in the $A_{e}$. We assume the determinants of these matrices are not identically 0 , and we consider the inverse

$$
N_{W^{\vee}}\left(A^{-1}\right)^{-1}=\left(b_{i j}\right)_{1 \leq i, j \leq n-m}
$$

It is easy to check using (4.3) that $b_{i j}=c_{i j} / \Psi_{H}$, where $c_{i j}$ is a homogeneous polynomial in the $A_{e}$ of degree $n+1$. In particular, the matrix (4.4) has entries homogeneous of degree 1 in the $A_{e}$.

The following is standard linear algebra.

Lemma 4.3. Let $q$ be a non-degenerate symmetric quadratic form on a vector space $V$ over $\mathbb{Q}$. Let $M$ be the invertible matrix representing $q$ with respect to some basis $\nu$ of $V$. Let $q^{\vee}$ denote the quadratic form on $V^{\vee}$ represented by $M^{-1}$ with respect to the dual basis $\nu^{\vee}$. Then $q^{\vee}$ is canonically defined independent of the choice of basis $\nu$.

For simplicity we now tensor with $\mathbb{Q}$ and write $W:=\mathbb{Q}[V]^{0}=$ $\left\{\sum n_{i} v_{i} \mid \sum n_{i}=0\right\}$.

Proposition 4.4. The momentum space $W$ of our graph has a canonical quadratic form $R\left(w_{1}, w_{2}\right)$ with coefficients rational functions in the $A_{e}$. For a suitable choice of basis, this quadratic form is given by the symmetric matrix (4.4).

We will see that the graph motive is independent of external momenta, but the period varies as a function of momenta.

Although $\Psi_{H}$ is defined for any $H \subset \mathbb{Q}[E]$, it has a particularly nice description when $H=H_{1}(\Gamma)$ for a graph $\Gamma$. A spanning tree $T \subset \Gamma$ for a connected graph $\Gamma$ is a connected and simply connected subgraph $T \subset \Gamma$ containing every vertex of $\Gamma$. (When $\Gamma$ itself is not connected, it is convenient to define a spanning tree to be the disjoint union of spanning trees in every connected component.) The following is classical (cf. [BEK], Prop. 2.2)

Proposition 4.5. With notation as above

$$
\Psi_{\Gamma}(A)=\sum_{T \subset \Gamma} \prod_{e \notin T} A_{e} .
$$

Here the sum is over all spanning trees $T \subset \Gamma$.

One last amusing point concerning the linear algebra of the graph polynomial: we can put metrics on the spaces of chains $\mathbb{Q}[E(\Gamma)]$ and $\mathbb{Q}[V(\Gamma)]$ and define the graph laplacian to be $d d^{*}$ :

$$
\mathbb{Q}[V]^{0} \subset \mathbb{Q}[V] \stackrel{d^{*}}{\rightarrow} \mathbb{Q}[E] \stackrel{d}{\rightarrow} \mathbb{Q}[V]^{0} \subset \mathbb{Q}[V] .
$$


Suppose our metrics are such that $\left\langle v_{i}, v_{j}\right\rangle=\delta_{i j}$ and $\left\langle e_{i}, e_{j}\right\rangle=A_{i} \cdot \delta_{i j}$. Write $d e_{j}=\sum_{i} a_{i j} v_{i}$. Then $d^{*} v_{i}=\sum_{j} a_{i j} A_{j}^{-1} e_{j}$ and

$$
d d^{*}\left(v_{i}\right)=\sum_{j, k} a_{i j} A_{j}^{-1} a_{k j} v_{k} .
$$

On the other hand, the matrix $N_{W^{\vee}}^{-1}\left(A^{-1}\right)$ above is obtained as follows. to each $e_{j}$ we associate the functional $\mathbb{Q}[V]^{\vee} \rightarrow \mathbb{Q}$ given by $\sum_{i} a_{i j} v_{i}$. The quadratic form associated to the square of this functional is

$$
\left\langle v_{\mu}, v_{\nu}\right\rangle=a_{\mu j} a_{\nu j}
$$

and the full matrix has $(\mu, \nu)$-entry $\sum_{j} A_{j}^{-1} a_{\mu j} a_{\nu j}$. Comparing with (4.7) and (4.3) we conclude:

Proposition 4.6. $\Psi_{\Gamma}=\prod_{e} A_{e} \cdot \operatorname{det} \operatorname{Laplacian}_{A}(\Gamma)$, where the notation means that the Laplacian is computed in the metric where each edge $e$ has length $A_{e}$.

\section{The Schwinger Trick}

The integral (3.11) looks like a period (i.e. the integral of an algebraic differential form over an homology cycle), but one does not see clearly the role of the graph $\Gamma$. Ultimately, we would like to relate the combinatorics of the graphs to the periods, so it is convenient to change the form of the period using the Schwinger trick.

Concretely, the Schwinger trick amounts to manipulating the integral (3.10) using the evident integral

$$
\int_{0}^{\infty} \exp (-q(Z) t) d t=1 / q(Z)
$$

I will not give the details (for a clear presentation, cf. [IZ], section 6-2-3), but let me say a few words about the final shape of the formula. In what follows $P \in W$ is a momentum vector (definition 4.1. Drop, for a moment, our assumption that the graph $\Gamma$ is $\log$ divergent and simply assume in (3.10) that $h_{1}(\Gamma)=n$ and $\# E(\Gamma)=m$. Let $M_{i}$ be the $4 n \times 4 n$ symmetric matrix associated to the quadric $q_{i}$. Note that $M_{i}=N_{i} \otimes I_{4}$, where $I_{4}$ is the $4 \times 4$ identity matrix and $N_{i}$ is $n \times n$ symmetric. Define

$$
N:=\sum_{1}^{m} A_{i} N_{i} ; \quad \Psi=\Psi_{\Gamma}:=\operatorname{det}(N) .
$$

Itzykson and Zuber (op. cit.) show that the Schwinger parametrization applied to a more general Feynman amplitude than (3.10) with nontrivial external momenta (to simplify, I have taken all masses $=0$. 
I have also done a Wick rotation to replace $i$ in the exponential with -1.) leads to an integral of the form:

$$
I_{\Gamma}(P):=\frac{1}{\left(16 \pi^{2}\right)^{n}} \int_{[0, \infty]^{m}} \frac{\exp \left(-P\left(N^{-1}\right)^{t} P\right)}{\Psi_{\Gamma}^{2}} d A_{1} \ldots d A_{m}
$$

The change of variable $A_{i}=\lambda B_{i}$ yields (with $R(P, P)$ as in Proposition

$$
I_{\Gamma}(P)=\frac{1}{\left(16 \pi^{2}\right)^{n}} \int_{A_{i} \geq 0, \sum A_{i}=1} \frac{\Omega}{\Psi^{2}} \int_{\lambda=0}^{\infty} \exp (-\lambda R(P, P)) \lambda^{m-1-2 n} d \lambda .
$$

To conclude, if we assume that $R(P, P)>0$ we can make the change of variable $\nu=\lambda R(P, P)$. Let $\sigma=\left\{\left(A_{1}, \ldots, A_{m}\right) \mid A_{i} \geq 0\right\} \subset \mathbb{P}^{m-1}(\mathbb{R})$. We can rewrite our integral (Note $\Gamma$ here is both the graph and the Gamma function. My apologies...)

$$
\begin{aligned}
I_{\Gamma}(P)= & \frac{1}{\left(16 \pi^{2}\right)^{n}} \int_{\sigma} \frac{R(P, P)^{2 n-m} \Omega}{\Psi^{2}} \int_{\nu=0}^{\infty} \exp (-\nu) \nu^{m-2 n} d \nu / \nu= \\
& \frac{\Gamma(2 n-m)}{(4 \pi)^{2 n}} \int_{\sigma} \frac{R(P, P)^{2 n-m} \Omega}{\Psi^{2}}=\frac{\Gamma(2 n-m)}{(4 \pi)^{2 n}} \int_{\sigma} \omega(P) .
\end{aligned}
$$

Here $\omega(P)=R(P, P)^{2 n-m} \Omega / \Psi^{2}$ represents a family of de Rham cohomology classes in $H_{D R}^{m-1}\left(\mathbb{P}^{m-1}-X_{\Gamma}\right)$, where $X_{\Gamma}: \Psi=0$ is the graph hypersurface.

Our strategy will be to define the motive of the graph $\Gamma$ to be

$$
M(\Gamma):=H^{m-1}\left(\mathbb{P}^{m-1}-X_{\Gamma}, \mathbb{Q}(m-1)\right) .
$$

Since the entries of the quadratic form $R$ are rational functions in the $A_{e}$ with denominator $\Psi$, we see that in the convergent case $2 n>m$, $\omega(P)$ in $(5.5)$ is a family of forms parametrized by external momentum $P$ representing classes in the de Rham cohomology of the motive. Although I do not pursue it here, it seems that a similar phenomenon occurs, e.g. when working in non-scalar theories. The motive remains $H^{m-1}\left(\mathbb{P}^{m-1}-X_{\Gamma}\right)$ depending only on the graph, but the period can vary.

In the log divergent case $m=2 n$ or the divergent case $2 n<m$, the $\Gamma$ factor in (5.5) contributes a pole, so the Feynman amplitude diverges, but we can still try to make sense of the residue term represented by $\omega(P)$. Note that in the log divergent case, $\omega=\omega(P)$ does not depend on external momenta. In the pure divergent case, $\omega(P)$ has a factor other than a power of $\Psi$ in the denominator, so it does not represent a class in the de Rham cohomology of the motive. 
Finally, we take a minute to consider the log divergent case $m=2 n$ from an algebro-geometric viewpoint. Let $\mathcal{Q}: A_{1} q_{1}+\ldots+A_{2 n} q_{2 n}=0$ be the universal quadric in the family spanned by the $q_{i}, 1 \leq i \leq 2 n$ :

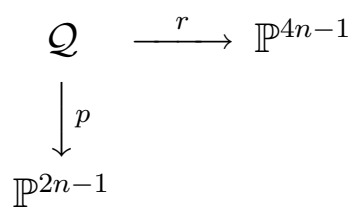

The fibres of $r$ are hyperplanes in $\mathbb{P}^{2 n-1}$ except along the locus $q_{1}=$ $\ldots=q_{2 n}=0$, where they jump to the whole of $\mathbb{P}^{2 n-1}$. Vertically, $p$ is a bundle of quadrics which degenerates over the zeroes of $\Phi\left(A_{1}, \ldots, A_{2 n}\right):=$ $\operatorname{det}\left(A_{1} M_{1}+\ldots+A_{2 n} M_{2 n}\right)$. Here $M_{i}$ is the $4 n \times 4 n$ symmetric matrix associated to the quadric $q_{i}$. One shows ([BEK], section 6 ) for some $c \in \mathbb{Q}^{\times}$

$$
\operatorname{Per}(q)=\frac{c}{\pi^{2 n}} \int_{\sigma} \frac{\Omega_{2 n-1}(A)}{\sqrt{\Phi}} ; \quad \sigma=\left\{\left(a_{1}, \ldots, a_{2 n}\right) \in \mathbb{P}^{2 n-1}(\mathbb{R}) \mid a_{i} \geq 0\right\}
$$

But in our case, the symmetric matrices all have the form $M_{i}=$ $N_{i} \otimes I_{4}$, where $I_{4}$ is the matrix for $x_{1}^{2}+\ldots+x_{4}^{2}$. Thus $\Phi=\Psi^{4}$ for a suitable $\Psi$ homogeneous of degree $n$, and we obtain

$$
\operatorname{Per}(\Gamma):=\operatorname{Per}(q)=\frac{c}{\pi^{2 n}} \int_{\sigma} \frac{\Omega_{2 n-1}(A)}{\Psi^{2}} ; \quad c \in \mathbb{Q}^{\times} .
$$

In the log divergent case, we will write

$$
\omega_{\Gamma}:=\frac{\Omega_{2 n-1}(A)}{\Psi^{2}} .
$$

\section{Combinatorial Structure of the Graph Hypersurface}

Clearly, to be able to say something about the period (5.9), we must know more about the hypersurface

$$
X=X_{\Gamma}: \Psi=0 .
$$

In this section we focus on the combinatorial aspect of the situation. We continue to assume $\Gamma$ has $m$ edges and $h_{1}(\Gamma)=n$. Notice that even when $m \leq 2 n$ so $\omega(P) \in M(\Gamma)_{D R}$, we cannot say that our period $\int_{\sigma} \frac{R(P, P) \Omega}{\Psi^{2}}$ is convergent, because the chain $\sigma$ can meet the polar locus $X_{\Gamma}$. Recall $\sigma$ is the set of points in $\mathbb{P}^{m-1}$ which can be represented in terms of the homogeneous coordinates $A_{e}$ with all entries real and nonnegative. Since our graph polynomial is a sum of terms with coefficient +1 (Proposition 4.5) we conclude 
Lemma 6.1. $\sigma \cap X_{\Gamma}=\bigcup_{L} \sigma_{L}$ where $L$ runs through all coordinate linear spaces $L: A_{e_{1}}=\ldots=A_{e_{p}}=0$ such that $L \subset X_{\Gamma}$, and $\sigma_{L}=$ $\sigma \cap L(\mathbb{R})$.

The following is a simple combinatorial exercise ([BEK], Prop. 3.1)

Lemma 6.2. A coordinate linear space $L: A_{e_{1}}=\ldots=A_{e_{p}}=0$ is contained in $X_{\Gamma}$ if and only if the union of the edges $e_{1} \cup \ldots \cup e_{p}$ supports a loop (i.e. writing $\Gamma_{L}$ for this subgraph (including all endpoints of the $\left.e_{i}\right)$, we have $h_{1}\left(\Gamma_{L}\right)>0$.)

Combining these two lemmas, we get a nice combinatorial description of $\sigma \cap X_{\Gamma}$ :

$$
\sigma \cap X_{\Gamma}=\bigcup_{L: h_{1}\left(\Gamma_{L}\right)>0} \sigma_{L}
$$

The idea will be to blow up $L \subset X_{\Gamma}$ in $\mathbb{P}^{m-1}$ and study the strict transform of $X_{\Gamma}$ in the blowup. The key point is

Proposition 6.3 ([BEK], Prop. 3.5). Let $\Gamma^{\prime} \subset \Gamma$ be a subgraph, and assume $h_{1}\left(\Gamma^{\prime}\right)>0$. Define the modified quotient graph $\Gamma / / \Gamma^{\prime}$ to be the graph obtained from $\Gamma$ by contracting each connected component of $\Gamma^{\prime}$ to a point. (Do not identify the points associated to different connected components.) We have Edge $(\Gamma)=E d g e\left(\Gamma^{\prime}\right) \amalg E d g e\left(\Gamma / / \Gamma^{\prime}\right)$. Suppose edge variables $A_{1}, \ldots, A_{r}$ are associated to $\Gamma^{\prime}$ and $A_{r+1}, \ldots, A_{m}$ to $\Gamma / / \Gamma^{\prime}$. Then the graph polynomials satisfy

$$
\Psi_{\Gamma}=\Psi_{\Gamma^{\prime}}\left(A_{1}, \ldots, A_{r}\right) \cdot \Psi_{\Gamma / / \Gamma^{\prime}}\left(A_{r+1}, \ldots, A_{m}\right)+F\left(A_{1}, \ldots, A_{m}\right)
$$

where the degree of $F$ in $A_{1}, \ldots, A_{r}$ is strictly greater than the degree of $\Psi_{\Gamma^{\prime}}\left(=h_{1}\left(\Gamma^{\prime}\right)\right)$.

This result can be interpreted geometrically as follows. Let $L=L_{\Gamma^{\prime}}$ : $A_{1}=\ldots=A_{r}=0$. By assumption $h_{1}\left(\Gamma^{\prime}\right)>0$ so from Lemma 6.2 we have $L \subset X_{\Gamma}$. Let $\pi: P \rightarrow \mathbb{P}^{m-1}$ be the blowup of $\mathbb{P}^{m-1}$ along $L$, and let $E, Y \subset P$ be the exceptional divisor and the strict transform of $X_{\Gamma}$, respectively. We have the diagram

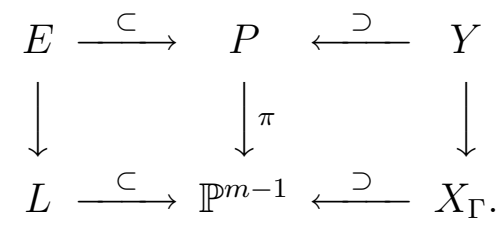

The normal bundle to $L$ in $\mathbb{P}^{m-1}$ is $\mathcal{O}_{L}(1)^{r}$, so

$$
E \cong \mathbb{P}\left(N_{L / \mathbb{P} m-1}\right) \cong L \times \mathbb{P}^{r-1}
$$


with homogeneous coordinates $\bar{A}_{i}, i=1, \ldots, r$ on $\mathbb{P}^{r-1}$ naturally associated to the coordinates $A_{1}, \ldots, A_{r}$ vanishing on $L$. Proposition 6.3 can be interpreted as saying

$$
\begin{gathered}
Y \cap E=\left(X_{\Gamma / / \Gamma^{\prime}} \times \mathbb{P}^{r-1}\right) \cup\left(L \times X_{\Gamma^{\prime}}\right) \\
E-Y \cap E=\left(L-X_{\Gamma / / \Gamma^{\prime}}\right) \times\left(\mathbb{P}^{r-1}-X_{\Gamma^{\prime}}\right) .
\end{gathered}
$$

In this way, blowing up cordinate linear spaces in $X_{\Gamma}$ is linked to subgraphs $\Gamma^{\prime} \subset \Gamma$ with $h_{1}\left(\Gamma^{\prime}\right)>0$. We shall also need to think about subgraphs $\Gamma^{\prime} \subset \Gamma$ (Forests) where $h_{1}\left(\Gamma^{\prime}\right)=0$. Again let $L: A_{1}=\ldots=$ $A_{r}=0$ be the coordinate linear space. In this case, the result is

$$
L \cap X_{\Gamma}=X_{\Gamma / / \Gamma^{\prime}} \subset L \cong \mathbb{P}^{m-r-1}
$$

Example 6.4. Let $\Gamma^{\prime}=e$ be a single edge with corresponding variable $A_{1}$. If $e$ meets two distinct vertices, then $X_{\Gamma} \cap\left\{A_{1}=0\right\}=X_{\Gamma / e}$. If $e$ is a loop (tadpole in physics lingo, I guess because physicists would tend to have an external edge attached to the sole vertex) then the hyperplane $\left\{A_{1}=0\right\} \subset X_{\Gamma}$ (so $X_{\Gamma}$ is reducible). In this case, $X_{\Gamma / e}$ is identified with the intersection of $\left\{A_{1}=0\right\}$ with the other component of $X_{\Gamma}$.

\section{The Motive}

We have defined (5.6) the motive $M(\Gamma)=H^{m-1}\left(\mathbb{P}^{m-1}-X_{\Gamma}, \mathbb{Q}(m-\right.$ 1)). In truth, however, this is just a building block for the motivic theory. When we want to talk about the period (5.9), we have to work relative to the simplex $\Delta: \prod A_{i}=0 \subset \mathbb{P}^{m-1}$ in order for our chain $\sigma$ to be an homology cycle. We are thus led to the relative motive or relative cohomology

$$
H^{m-1}\left(\mathbb{P}^{m-1}-X_{\Gamma}, \Delta-\Delta \cap X_{\Gamma}\right) .
$$

Note that the faces of $\Delta$ are the coordinate linear spaces $L$ as above. For such an $L: A_{e_{1}}=\ldots=A_{e_{p}}=0$, let $\Gamma_{L}=e_{1} \cup \ldots \cup e_{p} \subset \Gamma$. We have seen that $L \cap X_{\Gamma}$ is proper iff $h_{1}\left(\Gamma_{L}\right)=0$, in which case

$$
X_{\Gamma} \cap L \cong X_{\Gamma / / \Gamma_{L}}
$$

Conversely, those faces contained in $X_{\Gamma}$ correspond to subgraphs with $h_{1}>0$. These have to be blown up. If we just blow up one $L$ (would that life were so simple), the picture becomes as in (6.4). Our new candidate for the relative motive would be

$$
H^{m-1}(P-Y, B-Y \cap B, \mathbb{Q}(m-1)) ; \quad B=\pi^{*}(\Delta)
$$


We have added a new codim. 1 face $E$ by blowing up in $\mathbb{P}^{m-1}$ a higher codimension face $L \subset \Delta$. Motivically, by (6.6) we can identify

$$
H^{m-2}(E-Y \cap E, \mathbb{Q}(m-2))=M\left(\Gamma_{L}\right) \otimes M\left(\Gamma / / \Gamma_{L}\right) .
$$

Remark 7.1. We use the word motive rather casually. The reader loses nothing by thinking of étale or Betti cohomology instead. Indeed, implicitly in (7.4) we have used cohomological vanishing: viz. $H^{i}\left(\mathbb{P}^{m-1}-X_{\Gamma}, \mathbb{Q}(m-1)\right)=(0), i>m-1$ for any graph $\Gamma$ with $m$ edges. This is true cohomologically because $\mathbb{P}^{m-1}-X_{\Gamma}$ is affine. I do not know to what extent it is true in the motivic category.

As a consequence of (7.4), we get an important piece of structure, the comultiplication in the category of graph motives $M(\Gamma)$. This is not done in $[\mathrm{BEK}]$, so I give some technical details.

Construction 7.2. Let $\Gamma$ be a graph, and let $\Gamma^{\prime} \subset \Gamma$ be a subgraph. Assume $h_{1}\left(\Gamma^{\prime}\right)>0$. Then we have defined a residue map

$$
M(\Gamma) \rightarrow M\left(\Gamma^{\prime}\right) \otimes M\left(\Gamma / / \Gamma^{\prime}\right) .
$$

Proof. Let $\Gamma^{\prime}=e_{1} \cup \ldots \cup e_{p}$, and let $L: A_{1}=\ldots=A_{p}=0$. Let $\pi: P \rightarrow \mathbb{P}^{m-1}$ be the blowup of $L$, and let $Y \subset P($ resp. $E \subset P$ ) be the strict transform of $X_{\Gamma}$ (resp. the exceptional divisor). We have

$$
\mathbb{P}^{m-1}-X_{\Gamma}=P-Y-E \subset P-Y
$$

and hence a residue map

$$
\begin{aligned}
& M(\Gamma)=H^{m-1}\left(\mathbb{P}^{m-1}-X_{\Gamma}, \mathbb{Q}(m-1)\right) \\
& \quad \stackrel{\text { res }}{\longrightarrow} H^{m-2}(E-E \cap Y, \mathbb{Q}(m-2)) \stackrel{(7.4)}{\cong} M\left(\Gamma^{\prime}\right) \otimes M\left(\Gamma / / \Gamma^{\prime}\right) .
\end{aligned}
$$

Remark 7.3. This residue can be extended to the relative motive (7.1). I omit the details.

One other important property of our graph motives which is not quite as trivial as one might expect is multiplicativity under coproduct of graphs. Again details are not in the literature, so I give a sketch.

Proposition 7.4. Suppose $\Gamma=\Gamma_{1} \amalg \Gamma_{2}$ is a disjoint union of graphs. Then

$$
M(\Gamma) \cong M\left(\Gamma_{1}\right) \otimes M\left(\Gamma_{2}\right)
$$

Proof. Let $A_{i}^{(j)}$ be the edge variables for $\Gamma_{j}$. By definition, the graph polynomial of $\Gamma$ is

$$
\Psi_{\Gamma}\left(A^{(1)}, A^{(2)}\right)=\Psi_{\Gamma_{1}}\left(A^{(1)}\right) \cdot \Psi_{\Gamma_{2}}\left(A^{(2)}\right)
$$


Write $\mathbb{P}_{12}, \mathbb{P}_{1}, \mathbb{P}_{2}$ for the projective spaces with homogeneous coordinates $\left(A^{(1)}, A^{(2)}\right), A^{(1)}, A^{(2)}$ respectively. Define coordinate linear spaces $L_{1}, L_{2} \subset \mathbb{P}_{12}$ with $L_{j}: A^{(j)}=0$. Since the $L_{j} \subset X_{\Gamma}$, one has a diagram of $\mathbb{G}_{m}$-bundles

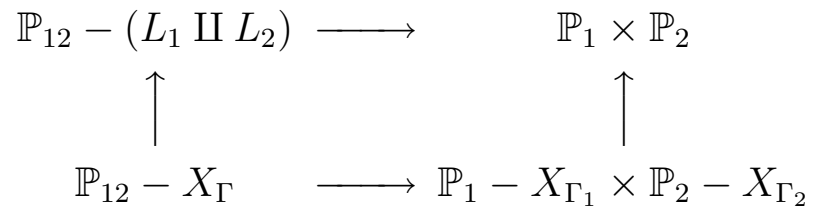

The proposition now follows from the observation that if $X, Y$ are affine varieties of dimensions $n, n-1$ respectively such that $X$ is a $\mathbb{G}_{m}$-bundle over $Y$, then $H^{n}(X, \mathbb{Q}(n)) \cong H^{n-1}(Y, \mathbb{Q}(n-1))$.

Construction 7.2 and Proposition 7.4 suggest that there is a Hopf algebra lurking somewhere.

Definition 7.5. A coproduct rule $\mathcal{S}$ is a rule associating to each graph $\Gamma$ a subset $\mathcal{S}(\Gamma) \subset 2^{E d g e(\Gamma)}$. We assume $\emptyset \in \mathcal{S}(\Gamma)$ and $\Gamma \in \mathcal{S}(\Gamma)$. Further, for subgraphs $\gamma_{2} \subset \gamma_{1} \subset \Gamma$, we require

$\gamma_{1} \in \mathcal{S}(\Gamma)$ and $\gamma_{2} \in \mathcal{S}\left(\gamma_{1}\right) \Leftrightarrow \gamma_{2} \in \mathcal{S}(\Gamma)$ and $\gamma^{\prime}:=\gamma_{1} / / \gamma_{2} \in \mathcal{S}\left(\Gamma / / \gamma_{2}\right)$

(Note, in passage from right to left, we are given $\gamma_{2}$ and $\gamma^{\prime}$, and we define $\gamma_{1}$ to be the inverse image in $\Gamma$ of $\gamma^{\prime} \subset \Gamma / / \gamma_{2}$. )

Proposition 7.6. Let $\mathcal{S}$ be a coproduct rule. Define, for a graph $\Gamma$

$$
\Delta_{\Gamma}=\oplus \operatorname{res}_{\Gamma^{\prime}}: M(\Gamma) \rightarrow \bigoplus_{\Gamma^{\prime} \in \mathcal{S}(\Gamma)} M\left(\Gamma^{\prime}\right) \otimes M\left(\Gamma / / \Gamma^{\prime}\right)
$$

Then $\Delta$ is coassociative in the sense that $(1 \otimes \Delta) \circ \Delta=(\Delta \otimes 1) \circ \Delta$.

Proof. The target of $(1 \otimes \Delta) \circ \Delta($ resp. $(\Delta \otimes 1) \circ \Delta)$ on $M(\Gamma)$ is

$$
\begin{gathered}
\bigoplus_{\Gamma^{\prime \prime} \in \mathcal{S}(\Gamma)} \bigoplus_{\Gamma^{\prime} \in \mathcal{S}\left(\Gamma / / \Gamma^{\prime \prime}\right)} M\left(\Gamma^{\prime \prime}\right) \otimes M\left(\Gamma^{\prime}\right) \otimes M\left(\left(\Gamma / / \Gamma^{\prime \prime}\right) / / \Gamma^{\prime}\right) \\
\text { resp. } \bigoplus_{\Gamma_{1} \in \mathcal{S}(\Gamma)} \bigoplus_{\Gamma_{2} \in \mathcal{S}\left(\Gamma_{1}\right)} M\left(\Gamma_{2}\right) \otimes M\left(\Gamma_{1} / \Gamma_{2}\right) \otimes M\left(\Gamma / / \Gamma_{1}\right) .
\end{gathered}
$$

The two objects coincide by the property of $\mathcal{S}$.

Examples 7.7. (i) The Hopf algebra of graphs was originally developed by Kreimer $[\mathrm{K}]$ as a successful attack on renormalization. Roughly speaking, $\mathcal{S}(\Gamma)$ was taken to be $\Gamma, \emptyset$ together with all divergent subgraphs, i.e. all subgraphs $\Gamma^{\prime}$ such that $\# E d g e\left(\Gamma^{\prime}\right) \leq 2 h_{1}\left(\Gamma^{\prime}\right)$. For this to work requires a physical theory where the collection of graphs is controlled by a physical Lagrangian. In the abstract graph setting of this paper, a renormalization comultiplication of this sort will not be 
coassociative. Indeed, if the graph $\Gamma$ is say log divergent $(\# E d g e(\Gamma)=$ $\left.2 h_{1}(\Gamma)\right)$ and if $\Gamma^{\prime} \subset \Gamma$ is worse than log divergent then the quotient $\Gamma / / \Gamma^{\prime}$ is convergent. This causes problems with coassociativity.

(ii) Define $\mathcal{S}(\Gamma)$ to be $\Gamma$ itself, together with all minimal $\Gamma^{\prime} \subset \Gamma$, where a graph $\Gamma^{\prime}$ is said to be minimal if for any $\Gamma^{\prime \prime} \subsetneq \Gamma^{\prime}$ we have $h_{1}\left(\Gamma^{\prime \prime}\right)<h_{1}\left(\Gamma^{\prime}\right)$. (Note $\emptyset$ is minimal for $h_{1}=0$.) Then $\mathcal{S}$ is a coproduct rule. (Exercise.) It is this coproduct which is relevant for constructing the motive, leaving questions of renormalization aside. Indeed it is shown in $[\mathrm{BEK}]$ that blowing up linear spaces corresponding to minimal subgraphs (starting with the maximal minimal subgraphs) suffices to separate $\sigma$ from the strict transform of $X_{\Gamma}$.

To actually explicit a Hopf algebra in the category of motives, one can proceed as follows. Fix a set $G r$ of graphs which is closed under the operations of taking sub and quotient graphs and also disjoint union. Define

$$
\mathcal{G}=\mathcal{G}(G r):=\bigoplus_{\Gamma \in G r} M(\Gamma)
$$

Then if we fix a coproduct rule, $\mathcal{G}$ becomes a Hopf algebra in the category of motives. For example, one could take $G r=G r(\Gamma)$ to be the smallest such set containing a given graph $\Gamma$. We write $\mathcal{G}(\Gamma)$ for the resulting Hopf algebra. Actually, of more interest to us than the full Hopf algebra will be the arrow $\Delta_{\Gamma}$ in Proposition 7.6.

\section{Motives and Graph Homology}

In the previous section we were concerned with links between the combinatorics of the graph $\Gamma$ and the algebraic geometry of $\mathbb{P}^{m-1}-$ $X_{\Gamma}$. Of course, the simplest example of what were were discussing is contracting an edge $e$ which is not a loop:

$$
M(\Gamma / e)=H^{m-2}\left(\left(\left\{A_{e}=0\right\} \cap\left(\mathbb{P}^{m-1}-X_{\Gamma}\right), \mathbb{Q}(m-2)\right) .\right.
$$

To study these faces, it is convenient to introduce a variant on our earlier motive:

$$
\begin{aligned}
& M^{0}(\Gamma):=H^{m-1}\left(\mathbb{P}^{m-1}-\Delta-X_{\Gamma}, \mathbb{Q}(m-1)\right)= \\
& H^{m-1}\left(\mathbb{G}_{m}^{m-1}-X_{\Gamma} \cap \mathbb{G}_{m}^{m-1}, \mathbb{Q}(m-1)\right) .
\end{aligned}
$$

(Note $\mathbb{P}^{m-1}-\Delta \cong \mathbb{G}_{m}^{m-1}$ is a torus.) We have residue maps

$$
\operatorname{res}_{e}: M^{0}(\Gamma) \rightarrow M^{0}(\Gamma / e) .
$$

We have $r e s_{e_{i}} \circ \operatorname{res}_{e_{j}}=-r e s_{e_{j}} \circ \operatorname{res}_{e_{i}}$ and hence a complex

$$
M^{0}(\Gamma) \stackrel{\partial}{\rightarrow} \bigoplus M^{0}(\Gamma / e) \stackrel{\partial}{\rightarrow} \bigoplus M^{0}\left(\Gamma / /\left\{e, e^{\prime}\right\}\right) \rightarrow \ldots
$$


This is a sort of motivic echo of graph homology as defined by Kontsevich [Ko1], [Ko2]. A number of variants are possible [CV], but the basic idea in graph homology is to consider the $\mathbb{Q}$-vector space of oriented graphs and to define a boundary operator by

$$
d[\Gamma]=\sum_{e} \pm[\Gamma / e]
$$

where $e \in \Gamma$ runs through all edges which are not loops. There are, however, at least three important distinctions. Firstly, we do not identify isomorphic pieces in our complex. It may happen that $\Gamma / /\left\{e_{i_{1}}, \ldots, e_{i_{p}}\right\} \cong \Gamma / /\left\{e_{j_{1}}, \ldots, e_{j_{p}}\right\}$ but the motives $M^{0}\left(\Gamma / /\left\{e_{i_{1}}, \ldots, e_{i_{p}}\right\}\right)$ and $M^{0}\left(\Gamma / /\left\{e_{j_{1}}, \ldots, e_{j_{p}}\right\}\right)$ are not identified in (8.4). This is because they correspond to distinct strata in the stratification of $\mathbb{P}^{n}-X_{\Gamma}$ an hence must be counted separately.

Secondly, an edge $e \in \Gamma$ forms a loop if and only if $\Psi_{\Gamma}=A_{e} \cdot \Psi_{\Gamma / e}$ is divisible by $A_{e}$. It follows that

$$
M^{0}(\Gamma)=M^{0}(\widetilde{\Gamma}) \otimes H^{1}\left(\mathbb{G}_{m}, \mathbb{Q}(1)\right)^{\otimes \delta} \cong M^{0}(\widetilde{\Gamma})
$$

where $\widetilde{\Gamma}$ is the graph obtained from $\Gamma$ by shrinking the $\delta$ looped edges to points. In particular, $M^{0}(\Gamma)$ does not determine $h_{1}(\Gamma)$.

Finally, we do not need to orient our graphs. The complex (8.4) is defined independent of orientation.

W can now define with $G r$ as in (7.13)

$$
\mathcal{G}^{0}=\bigoplus_{\Gamma \in G r} M^{0}(\Gamma)
$$

where the sum now is over $\Gamma \in G r$ without looped edges. The multiplication and comultiplication structures carry over from $\mathcal{G}$, (7.13), so if we fix a comultiplcation rule, then $\mathcal{G}^{0}$ is a Hopf algebra. The comultiplication and the differential are both given by residues, so they anti-commute as remarked above. Thus

Theorem 8.1. With notation as above, $\mathcal{G}^{0}$ is a differential Hopf algebra.

Using the comultiplication and the derivation on $\mathcal{G}^{0}(\Gamma)$ we can build a double complex as follows. First, if $A$ is an associative coalgebra with comultiplication map $\Delta$, we have a complex

$$
A \stackrel{\Delta}{\longrightarrow} A \otimes A \stackrel{\Delta \otimes 1-1 \otimes \Delta}{\longrightarrow} A^{\otimes 3} \rightarrow \ldots
$$


If the comultiplication is compatible with a differential on $A$, we get a double complex. In our situation, this looks like

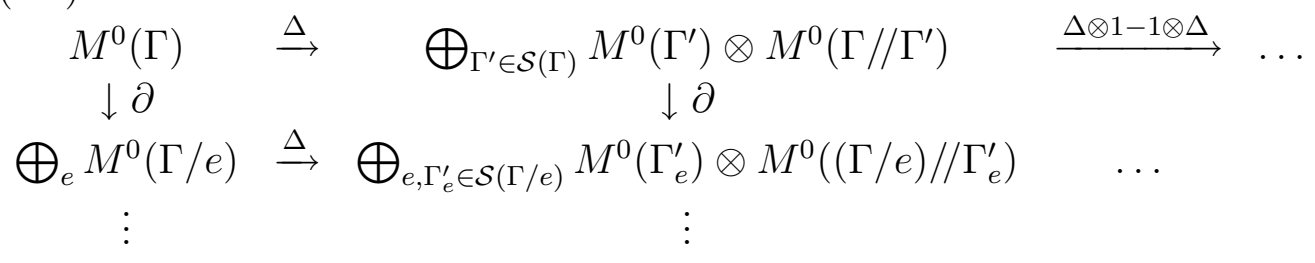

This differential Hopf algebra is related to the motive as follows. Recall, we started with $\mathbb{P}^{n}-X_{\Gamma}$ and then we blew up in $\mathbb{P}^{n}$ certain coordinate linear spaces contained in $X_{\Gamma}$. Quite generally, given a finite collection $\mathcal{L}$ of linear spaces in $\mathbb{P}^{n}$ which is closed under intersection, then there is a simple algorithm for blowing up to achieve a normal crossings divisor, [ESV]. One blows up the linear spaces in $\mathcal{L}$ which are minimal. These are necessarily disjoint. One takes the strict transforms of the remaining elements in $\mathcal{L}$ and again blows up the minimal elements, etc. We apply this with $\mathcal{L}$ the set of coordinate linear spaces contained in $X_{\Gamma}$. Let $\pi: P \rightarrow \mathbb{P}^{n}$ be the resulting scheme, and let $Y \subset P$ be the strict transform of $X_{\Gamma}$. $P$ has a normal crossings divisor $B=\pi^{*}(\Delta)$ where $\Delta$ is the coordinate simplex in $\mathbb{P}^{n}$, and no stratum of $B$ lies in $Y$. Using $B$, we can stratify $P-Y$ in the usual way, so

$$
(P-Y)^{(0)}=P-Y-B ; \quad(P-Y)^{(i)}=B_{(i)}-B_{(i+1)}-Y \cap B_{(i)}
$$

where $B_{(i)}$ denotes the closed stratum of $B$ of dimension $n-i$.

Associated to this stratification we have a spectral sequence

$$
E_{1}^{p, q}=H^{q-p}\left((P-Y)^{(p)}, \mathbb{Q}(n-p)\right) \Rightarrow H^{p+q}(P-Y, \mathbb{Q}(n)) .
$$

Proposition 8.2. The simple complex associated to the double complex (8.9) is the $E_{1}$ complex with $q=n$ in (8.11).

Note however that even in simple examples like the wheel with three spokes, one needs more in the spectral sequence than just the piece $q=n$ in order to calculate $M(\Gamma)$. This suggests that $M^{0}(\Gamma)$ should be replaced with some richer object like $R \Gamma\left(\mathbb{P}^{m-1}-X_{\Gamma}-\Delta, \mathbb{Q}(m-1)\right)$.

To simplify, write $T^{r}:=\mathbb{P}^{r}-\Delta \cong \mathbb{G}_{m}^{r}$ and $Y_{\Gamma}$ rather than $X_{\Gamma} \cap T^{m-1}$. The rest of this section is devoted to some tricks which will be used in the next section to calculate $H^{*}\left(T^{m-1}-Y_{\Gamma}\right)$ for graphs $\Gamma$ which occur as subgraphs in the wheel with three spokes.

The first trick concerns plane graphs. A graph $\Gamma$ is a plane graph if it can be drawn without crossings on the Riemann sphere $S^{2}$. If $\Gamma$ is a plane graph, then so is any face of $\Gamma$. The wheel with $n$ spokes is a plane graph. If $\Gamma$ is a connected plane graph, the dual graph $\Gamma^{\vee}$ 
is defined as follows. Embed $\Gamma$ in $S^{2}$. Choose one point $v_{i}$ in each component $D_{i}$ of $S^{2}-\Gamma$. These are the vertices of $\Gamma^{\vee}$. Draw one edge connecting $v_{i}$ and $v_{j}$ for every edge $e$ of $\Gamma$ such that $D_{i}$ and $D_{j}$ are connected by an arc meeting $e$ and no other edge of $\Gamma$. In particular, edges of $\Gamma$ and $\Gamma^{\vee}$ are in $1-1$ correspondence. For an example, see fig. 1 , where $\Gamma$ has the light edges and $\Gamma^{\vee}$ the dark.

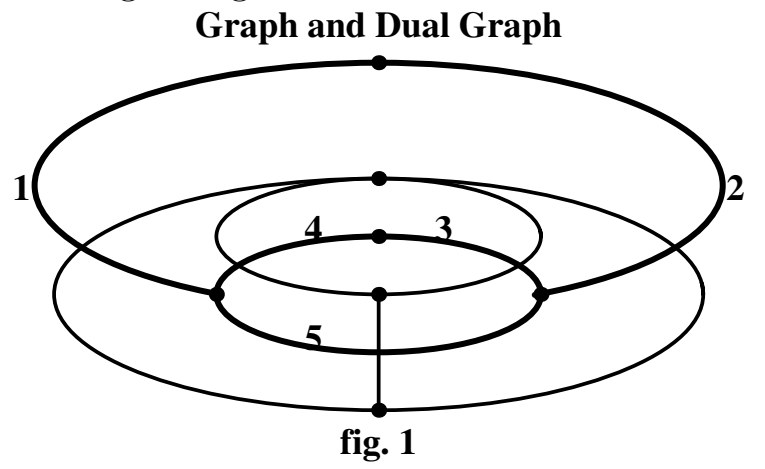

One has $\left(\Gamma^{\vee}\right)^{\vee}=\Gamma$. The dual of the banana graph with $n$ edges and 2 vertices (fig. 2) is the circle graph (fig. 3) with $n$ edges connected in a circle.
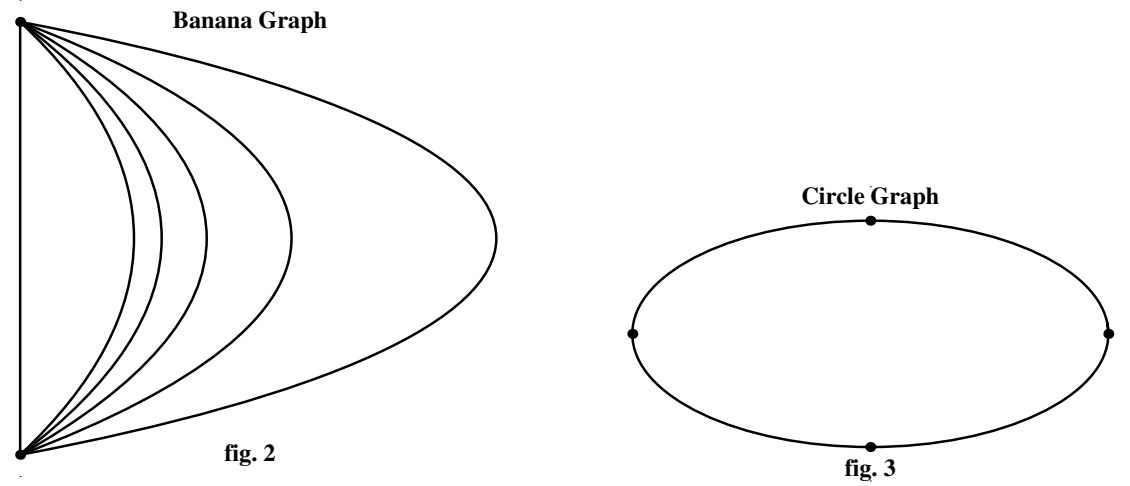

One checks

$$
\begin{gathered}
\# \text { Conn. } \operatorname{Comps}\left(S^{2}-\Gamma\right)=h_{1}(\Gamma)+1=\# \operatorname{Vertices}\left(\Gamma^{\vee}\right) \\
\# \operatorname{Edge}(\Gamma)=\# \operatorname{Edge}\left(\Gamma^{\vee}\right)
\end{gathered}
$$

Proposition 8.3. Let $\Gamma$ be a plane graph with $n+1$ edges. Let $A_{i}$ be the edge coordinates, and write $a_{i j}:=A_{i} / A_{j}$ for the functions on $T^{n}$. Then $a_{i j} \mapsto a_{j i}$ induces an involution of $T^{n}$ which carries $Y_{\Gamma} \cong Y_{\Gamma^{\vee}}$. 
Proof. This follows from the identity on graph polynomials

$$
\begin{aligned}
& \Psi_{\Gamma}(A)=\sum_{T \subset \Gamma} \prod_{e \notin T} A_{e}=\left(\prod_{e} A_{e}^{-1}\right) \sum_{T \subset \Gamma} \prod_{e \in T} A_{e}^{-1}= \\
& \sum_{T^{\vee} \subset \Gamma^{\vee}} \prod_{e \notin T^{\vee}} A_{e}^{-1}=\Psi_{\Gamma^{\vee}}\left(A^{-1}\right) .
\end{aligned}
$$

To understand (8.14), let $T \subset \Gamma$ be a spanning tree. Then $\Gamma / T$ is a plane graph with one vertex, i.e. a union of looped edges. Then $(\Gamma / T)^{\vee}$ is a tree which is a spanning tree in $\Gamma^{\vee}$.

Let $\Gamma$ be the circle graph with $n+1$ edges. Then $\Psi_{\Gamma}=A_{1}+\ldots+A_{n+1}$, so $T^{n}-Y_{\Gamma}=\mathbb{P}^{n}-\Delta-\left\{\sum A_{i}=0\right\}$ is a configuration which is the complement in $\mathbb{P}^{n}$ of a union of linear hyperplanes meeting transversally. The following is then well known:

Proposition 8.4. (i) $H^{1}\left(T^{n}-Y_{\Gamma}, \mathbb{Q}\right) \cong \mathbb{Q}(-1)^{\oplus n+1}$. (ii) $H^{*}\left(T^{n}-Y_{\Gamma}, \mathbb{Q}\right) \cong \bigwedge^{*} H^{1} / \bigwedge^{n+1} H^{1}$.

Definition 8.5. I will say a graph $\Gamma$ has linear cohomology if $H^{*}\left(T^{n}-\right.$ $\left.Y_{\Gamma}, \mathbb{Q}\right)$ is generated by the classes $\frac{1}{2 \pi i} d u_{j} / u_{j}, 1 \leq j \leq n+\nu$ where $u_{1}, \ldots, u_{n}$ are the standard units on $T^{n}$ and $u_{n+1}, \ldots, u_{n+\nu}$ are defining equations for the irreducible components of $Y_{\Gamma}$.

Remarks 8.6. (i) One can (and one should) go further by expliciting the relations among the generators (cf. [OT], Chap. 3 and Chap. 5.4. The full condition should be that $H^{*}\left(T^{n}-Y_{\Gamma}, \mathbb{Q}\right)$ forms an OrlikSolomon algebra, but I am uncertain precisely how to formulate this in our context.

(ii) If $\Gamma$ has linear cohomology then $H^{p}\left(T^{n}-Y_{\Gamma}, \mathbb{Q}\right)$ is pure of weight $2 p$.

(iii) Of course the obvious way for $\Gamma$ to have linear cohomology is to have an isomorphism $T^{n}-Y_{\Gamma} \cong T^{n}-\mathcal{L}$ where $\mathcal{L}$ is a finite set of linear hyperplanes. Note that such an isomorphism need not be linear.

(iv) Circle graphs have linear cohomology.

(v) Banana graphs (cf. fig. 2) have linear cohomology. Indeed, banana graphs are dual to circle graphs, so we can use Proposition 8.3.

(vi) We will see in the next section that all the graphs associated to non-trivial faces in the wheel with three spokes have linear cohomology.

Lemma 8.7. Let $\Gamma$ be a graph, and let $e \in \Gamma$ be an edge. Let $\Gamma^{\prime}$ be obtained from $\Gamma$ by subdividing e. Assume $\Gamma$ and $\Gamma / e$ have linear cohomology. Then $\Gamma^{\prime}$ has linear cohomology.

Proof. Assume $\Gamma$ has $n$ edges, and enumerate the edge variables $A_{1}, \ldots, A_{n}$ so that $e \leftrightarrow A_{n}$. Write $a_{i}=A_{i} / A_{1}$. We identify $T^{n-1}=\operatorname{Spec} \mathbb{Q}\left[a_{i}, a_{i}^{-1}\right]$. 
Let $A_{j}^{\prime}$ be the edge variables of $\Gamma^{\prime}$, and write $a_{j}^{\prime}=A_{j}^{\prime} / A_{1}^{\prime}$. On the level of the projective graph hypersurface, one can show (exercise) the map $A_{i} \mapsto A_{i}^{\prime}, i \leq n-1, A_{n} \mapsto A_{n}^{\prime}+A_{n+1}^{\prime}$ identifies $X_{\Gamma^{\prime}}$ with the cone over $X_{\Gamma}$.

The picture for $Y_{\Gamma}$ is a bit more complicated. Let $D^{\prime} \subset T^{n}$ be defined by $a_{n}^{\prime}+a_{n+1}^{\prime}=0$. Then we have

$$
\pi: T^{n}-Y_{\Gamma^{\prime}}-D^{\prime} \rightarrow T^{n-1}-Y_{\Gamma}
$$

In terms of coordinate rings

$$
\mathbb{Q}\left[a_{i}^{\prime}, a_{i}^{\prime-1}, \frac{1}{a_{n}^{\prime}+a_{n+1}^{\prime}}, \frac{1}{\psi_{\Gamma^{\prime}}}\right]=\mathbb{Q}\left[a_{i}, a_{i}^{-1}, \frac{1}{\psi_{\Gamma}}\right]\left[a_{n+1}^{\prime}, \frac{1}{a_{n+1}^{\prime}}, \frac{1}{a_{n}-a_{n+1}^{\prime}}\right]
$$

Since $a_{n}$ is a unit, it follows that

$$
T^{n}-Y_{\Gamma^{\prime}}-D^{\prime} \cong\left(T^{n-1}-Y_{\Gamma}\right) \times\left(\mathbb{G}_{m}-\{1\}\right) .
$$

By Künneth, we see that $H^{1}\left(T^{n}-Y_{\Gamma^{\prime}}-D^{\prime}, \mathbb{Q}\right) \cong \mathbb{Q}(-1)^{n+2}$ and that $H^{*}\left(T^{n}-Y_{\Gamma^{\prime}}-D^{\prime}, \mathbb{Q}\right)$ is generated as an algebra by $H^{1}$.

Similarly,

$$
\begin{aligned}
& D^{\prime} \cong \operatorname{Spec} \mathbb{Q}\left[a_{2}, a_{2}^{-1}, \ldots, a_{n-1}, a_{n-1}^{-1}, \frac{1}{\psi_{\Gamma / e}}\right]\left[a_{n}^{\prime}, \frac{1}{a_{n}^{\prime}}\right] \\
& \cong\left(T^{n-2}-Y_{\Gamma / e}\right) \times \mathbb{G}_{m} .
\end{aligned}
$$

By our asumptions, it follows that $H^{1}\left(D^{\prime}\right) \cong \mathbb{Q}(-1)^{n}$ generated by the classes of the evident units, and further that $H^{*}\left(D^{\prime}\right)$ is generated by $H^{1}$. In particular, $H^{1}\left(T^{n}-Y_{\Gamma^{\prime}}\right) \rightarrow H^{1}\left(D^{\prime}\right)$ and res $: H^{1}\left(T^{n}-Y_{\Gamma^{\prime}}-D^{\prime}, \mathbb{Q}\right) \rightarrow$ $H^{1}\left(D^{\prime}, \mathbb{Q}(-1)\right)$. This implies that the residue map is surjective in all degrees, so we get an exact sequence

$$
\begin{aligned}
0 \rightarrow H^{*}\left(T^{n}-Y_{\Gamma^{\prime}}, \mathbb{Q}\right) \rightarrow H^{*}\left(T^{n}-Y_{\Gamma^{\prime}}-D^{\prime}, \mathbb{Q}\right) \rightarrow \\
H^{*-1}\left(D^{\prime}, \mathbb{Q}(-1)\right) \rightarrow 0 .
\end{aligned}
$$

The assertion of the lemma follows from (8.18) and (8.19).

\section{The Wheel With 3 Spokes}

One knows from [So] that $\zeta(2 r+1)$ is the period associated to a motivic extension (that is, an extension which can be constructed geometrically)

$$
0 \rightarrow \mathbb{Q}(0) \rightarrow E \rightarrow \mathbb{Q}(-1-2 r) \rightarrow 0
$$

By [BK1], the Feynman amplitude associated to the wheel with $n$ spokes (fig. 4) is an elementary factor times $\zeta(2 n-3)$. 


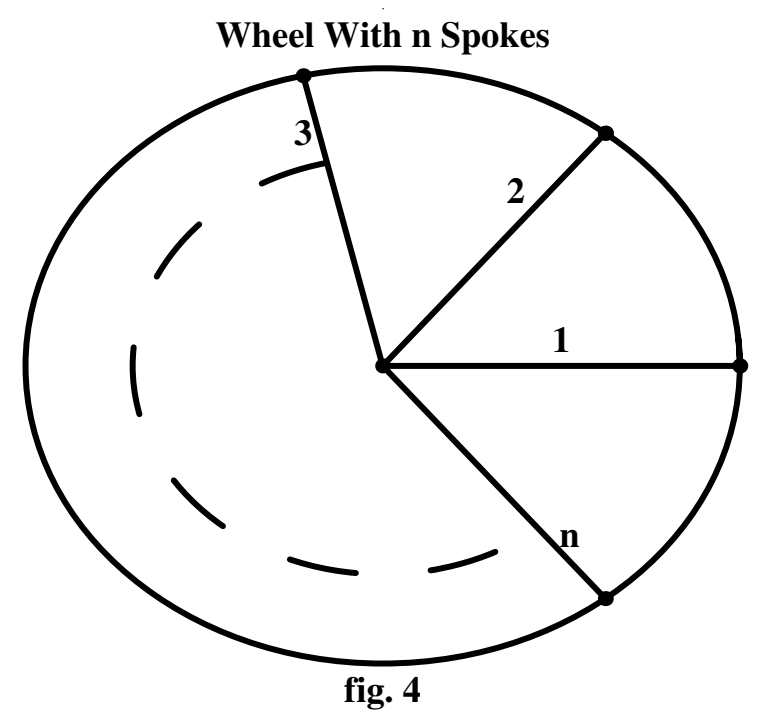

We have seen that this Feynman amplitude is a period for a motive of the form $H^{2 n-1}(P-Y, B-B \cap Y)$ where $P$ is some blowup of $\mathbb{P}^{2 n-1}$ and $Y$ is the strict transform of the graph hypersurface $X_{n}$. To get an extension of the form (9.1), we should construct arrows

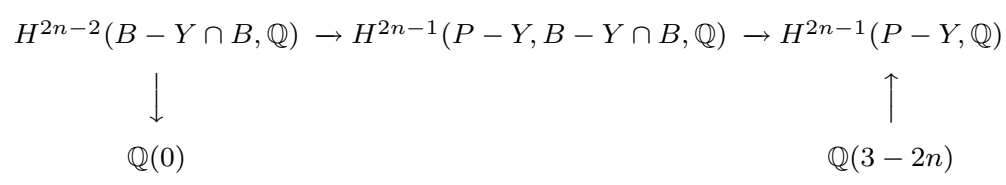

in such a way that the pushout and pullback are defined and yield an extension of the form (9.1). At the moment, the existence of such arrows is purely conjectural. Our main result ([BEK], Thm. 11.2) is

Theorem 9.1. Let $M_{n}:=H^{2 n-1}\left(\mathbb{P}^{2 n-1}-X_{n}, \mathbb{Q}(2 n-1)\right)$ be the motive associated to the wheel with $n$ spokes graph $\Gamma_{n}$. Then $M_{n} \cong \mathbb{Q}(2)$.

The proof is too complicated to give here. Note that it fits with (9.2) in the sense that the inclusion $\mathbb{P}^{2 n-1}-X_{n} \subset P-Y$ will give a map $H^{2 n-1}(P-Y, \mathbb{Q}) \rightarrow \mathbb{Q}(3-2 n)$, but this is not sharp enough to deduce what we want.

The case of the wheel with 3 spokes is rather exceptional. By way of example, I propose to work out some of the structure of the corresponding motive. Theorem 9.1 in the case $n=3$ is easier. First, note that $H_{1}\left(\Gamma_{3}\right)=\mathbb{Q}^{3}$. If the edges are labeled and oriented as in fig. 5 below 


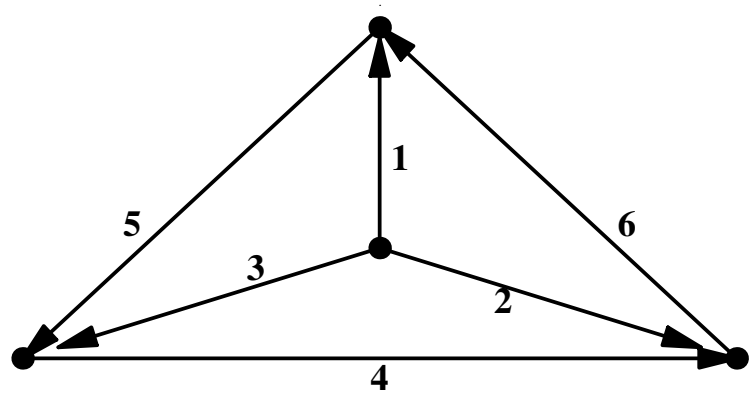

fig. 5

a basis for $H_{1}$ is given by

$$
f_{1}=e_{1}-e_{6}-e_{2} ; \quad f_{2}=e_{2}-e_{4}-e_{3} ; \quad f_{3}=e_{3}-e_{5}-e_{1} .
$$

From this one easily computes the rank 1 quadrics $\left.\left(e_{i}^{\vee}\right)^{2}\right|_{H_{1}\left(\Gamma_{3}\right)}$. For example,

$$
\left(e_{1}^{\vee}\right)^{2}=\left(\begin{array}{ccc}
1 & 0 & -1 \\
0 & 0 & 0 \\
-1 & 0 & 1
\end{array}\right) .
$$

In particular, one checks that the 6 quadrics $\left(e_{i}^{\vee}\right)^{2}$, viewed as homogeneous quadrics on $\mathbb{P}^{2}=\mathbb{P}\left(H_{1}\left(\Gamma_{3}\right)\right)$, span the complete linear system $\Gamma\left(\mathbb{P}^{2}, \mathcal{O}_{\mathbb{P}^{2}}(2)\right)$. We can identify $\mathbb{P}^{5}$ with the projective space of these quadrics, and $X_{3} \subset \mathbb{P}^{5}$ is the space of singular quadrics. Now a singular quadric on $\mathbb{P}^{2}$ is a union of 2 (possibly coincident) lines. The lines on $\mathbb{P}^{2}$ are parametrized by another $\mathbb{P}^{2}$, so we may identify

$$
X_{3} \cong \operatorname{Sym}^{2}\left(\mathbb{P}^{2}\right)
$$

The rank 1 quadrics correspond to double lines, and these are parametrized by the diagonal in $\operatorname{Sym}^{2}\left(\mathbb{P}^{2}\right)$.

Lemma 9.2. We have with $\ell:=c_{1}(\mathcal{O}(1) \otimes \mathcal{O}(1))$

$$
H^{4}\left(X_{3}, \mathbb{Q}\right) \cong H^{4}\left(\mathbb{P}^{2} \times \mathbb{P}^{2}, \mathbb{Q}\right)^{\mathbb{Z} / 2 \mathbb{Z}}=\mathbb{Q} \cdot\left[2 \mathbb{P}^{2} \times\{p t\}+2\{p t\} \times \mathbb{P}^{2}-\Delta\right] \oplus \mathbb{Q} \cdot \ell^{2}
$$

As Hodge structures,

$$
H^{4}\left(X_{3}, \mathbb{Q}\right) \cong \mathbb{Q}(-1)^{2} ; \quad H^{5}\left(\mathbb{P}^{5}-X_{3}, \mathbb{Q}\right) \cong \mathbb{Q}(-3) .
$$

Proof. Let $\pi: \mathbb{P}^{2} \times \mathbb{P}^{2} \rightarrow \operatorname{Sym}^{2}\left(\mathbb{P}^{2}\right)$. In the classical topology, one can define a trace map $\pi_{*} \mathbb{Q}_{\mathbb{P}^{2} \times \mathbb{P}^{2}} \rightarrow \mathbb{Q}_{\mathrm{Sym}^{2}\left(\mathbb{P}^{2}\right)}$ by simply adding up over the stalks. This defines a trace map $\pi_{*}: H^{*}\left(\mathbb{P}^{2} \times \mathbb{P}^{2}, \mathbb{Q}\right)=$ $H^{*}\left(\operatorname{Sym}^{2}\left(\mathbb{P}^{2}\right), \pi_{*} \mathbb{Q}\right) \rightarrow H^{*}\left(\operatorname{Sym}^{2}\left(\mathbb{P}^{2}\right), \mathbb{Q}\right)$, and $\pi_{*} \circ \pi^{*}$ is multiplication by 2 . This proves the lefthand isomorphism in (9.6). For the Hodge 
structure, note by duality $H^{5}\left(\mathbb{P}^{5}-X_{3}, \mathbb{Q}\right) \cong H_{c}^{5}\left(\mathbb{P}^{5}-X_{3}, \mathbb{Q}(5)\right)^{\vee}$. We conclude using the exact sequence

$$
0 \rightarrow H^{4}\left(\mathbb{P}^{5}\right) \rightarrow H^{4}\left(X_{3}\right) \rightarrow H_{c}^{5}\left(\mathbb{P}^{5}-X_{3}\right) \rightarrow 0 .
$$

Another computation of $H^{*}\left(\mathbb{P}^{5}-X_{3}\right)$, due to Deligne, is to remark that

$$
P S L_{3}(\mathbb{C}) / S O_{3}(\mathbb{C}) \cong \mathbb{P}^{5}-X_{3} ; \quad M \mapsto M \cdot{ }^{t} M
$$

Even in this case I cannot construct the arrows in (9.2). What is true, however, is that the locally closed strata of $B-Y \cap B$ are of a quite simple sort. They have linear cohomology in the sense of Definition 8.5 .

Recall we call a subgraph $G \subset \Gamma_{3}$ minimal if removing any edge from $G$ lowers $h_{1}$. The minimal subgraphs of $\Gamma_{3}$ are of three types.
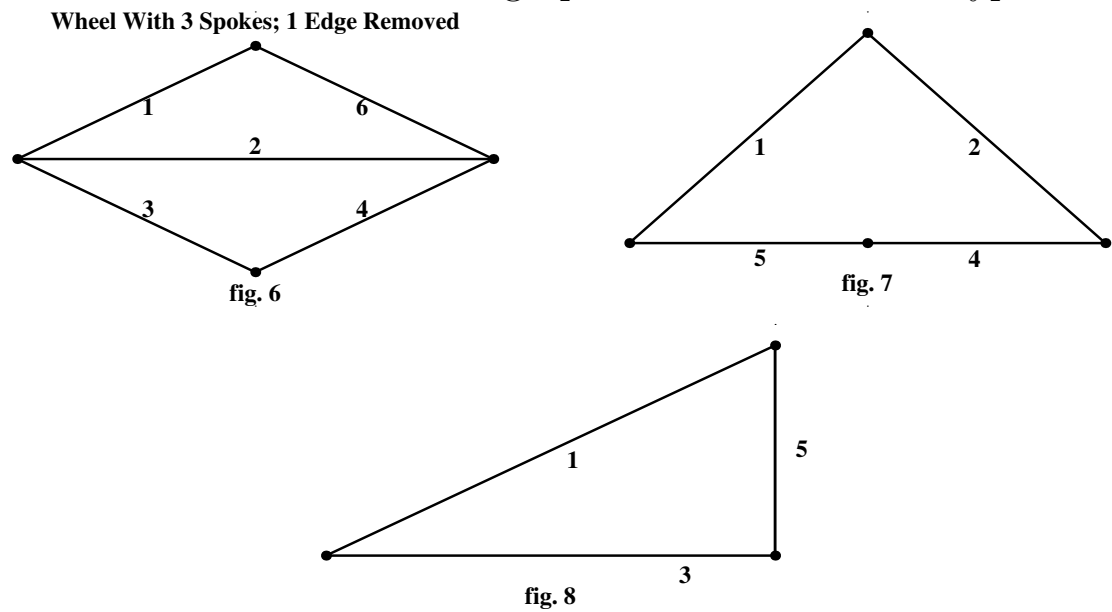

There are 6 subgraphs as in fig. 6 which are obtained by deleting an edge from $\Gamma_{3}$. In each case, the locus where the edge variables are zero is a point $(0, \ldots, 1, \ldots, 0) \in X_{3}$. Then there are 6 subgraphs as in fig. 7 obtained by removing 2 non-adjacent edges, and 4 subgraphs as in fig. 8 obtained by removing all the edges through a given vertex. The corresponding coordinate linear spaces have dimensions 1 and 2 respectively and are contained in $X_{3}$. A moment's thought convinces you that the union of two minimal subgraphs in this case is either the whole graph, in which case the two linear spaces are disjoint, or is a subgraph as in fig. 6, in which case the two linear spaces meet in a single coordinate vertex $(0, \ldots, 1, \ldots, 0)$. It follows that blowing up the 6 coordinate vertices makes the strict transforms of the other 10 minimal subgraphs disjoint. We Then blow them up in any order we like. 
To understand the picture, consider generally linear spaces

$$
\begin{gathered}
\mathbb{P}^{s} \subsetneq \mathbb{P}^{r} \subsetneq \mathbb{P}^{n}\left(A_{1}, \ldots, A_{n+1}\right) \\
\mathbb{P}^{s}: A_{1}=\ldots=A_{n-s}=0 ; \quad \mathbb{P}^{r}: A_{1}=\ldots=A_{n-r}=0
\end{gathered}
$$

Consider the effect of first blowing up $\mathbb{P}^{s} \subset \mathbb{P}^{n}$ and then blowing up the strict transform of $\mathbb{P}^{r}$. The picture looks like

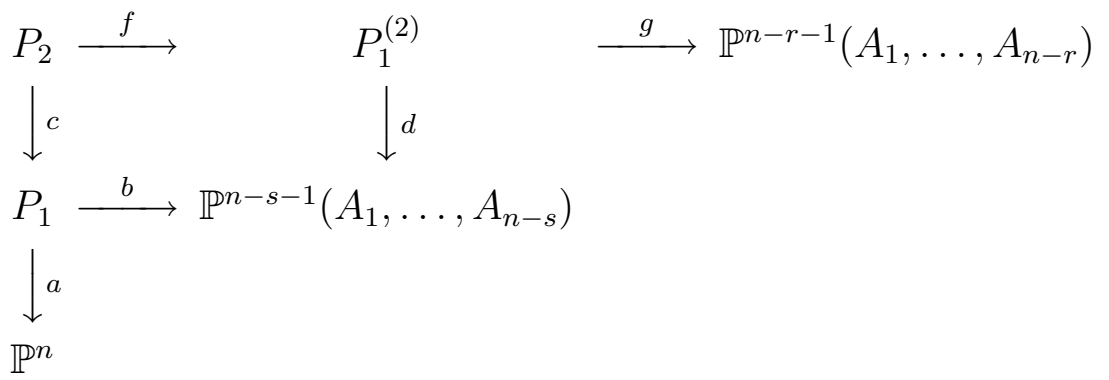

Here the maps $a$ and $d$ are blowups and $b$ and $g$ are given by projections

$$
\left(A_{1}, \ldots, A_{n+1}\right) \mapsto\left(A_{1}, \ldots, A_{n-s}\right) \mapsto\left(A_{1}, \ldots, A_{n-r}\right) .
$$

There are exceptional divisors $E_{1}^{(1)} \cong P^{s} \times P^{n-s-1} \subset P_{1}$ and $E_{1}^{(2)} \cong$ $\mathbb{P}^{r-s-1} \times \mathbb{P}^{n-r-1} \subset P_{1}^{(2)}$. The maps $\left.b\right|_{E_{1}^{(1)}}: E_{1}^{(1)} \rightarrow \mathbb{P}^{n-s-1}$ and $\left.g\right|_{E_{1}^{(2)}}:$ $E_{1}^{(2)} \rightarrow \mathbb{P}^{n-r-1}$ coincide with the projections. We define $E_{1}=c^{-1} E_{1}^{(1)}, E_{2}=$ $f^{-1} E_{1}^{(2)} \subset P_{2}$ and $Z=b^{-1}\left\{A_{1}=\ldots=A_{n-r}=0\right\} \subset P_{1}$. Then $Z$ is the strict transform in $P_{1}$ of $\mathbb{P}^{r}$ and $c$ is the blowup of $Z$. The square $b c=d f$ is cartesian.

Let $\pi:=a \circ c: P_{2} \rightarrow \mathbb{P}^{n}$. Inside $P_{2}$ we have a normal crossings divisor $B$ whose components are the strict transforms of the coordinate hyperplanes $\left\{A_{i}=0\right\}$ together with $E_{1}, E_{2}$. Note that $P_{2}$ has a toric structure, and the locally closed strata of $B$ are all tori with canonical coordinates. If $\Gamma$ is a graph with graph hypersurface $X_{\Gamma} \subset \mathbb{P}^{n}$ and strict transform $Y \subset P_{2}$, then the locally closed strata of $B-Y \cap B$ are all of the form $\prod_{i} M^{0}\left(\gamma_{i}\right)$ where $\gamma$ is a subquotient graph of $\Gamma$.

Let us apply this in the case of $X_{3} \subset \mathbb{P}^{5}$. In this case $s=0$ and we should consider separately the cases $r=1$ and $r=2$. For $r=1$ we have $\mathbb{P}^{0} \subset \mathbb{P}^{1} \subset \mathbb{P}^{5}$. In this case, $E_{1} \cap E_{2}=\mathbb{P}^{3}$ which one should perhaps identify with $\mathbb{P}^{0} \times \mathbb{P}^{0} \times \mathbb{P}^{3}$. It corresponds to the filtration on $\Gamma_{3}$ given by

$$
\left\{\Gamma_{3}-\text { two non-adjacent edges }\right\} \subset\left\{\Gamma_{3} \text { - one edge }\right\} \subset \Gamma_{3}
$$

Taking successive quotients yields two tadpoles (with graph hypersurfaces the empty set in $\mathbb{P}^{0}$ ) and one circle graph as in fig. 7. The circle 
graph has graph polynomial given by the sum of the graph coordinates, so we get $Y \cap E_{1} \cap E_{2}: A_{1}+\ldots+A_{4}=0$ in $\mathbb{P}^{3}$ for suitable coordinates.

The case $E_{1} \cap E_{2}$ for $r=2$ yields $\left(\mathbb{P}^{0}\right) \times \mathbb{P}^{1} \times \mathbb{P}^{2}$. The filtration on graphs is

(9.13) $\left\{\Gamma_{3}-\right.$ three edges through a vertex $\} \subset\left\{\Gamma_{3}-\right.$ one edge $\} \subset \Gamma_{3}$

We get two circles (one with three edges, the other with two) and one tadpole. $P$ is obtained from $\mathbb{P}^{5}$ by blowing up 16 times, so our divisor $B \subset P$ has 22 irreducible components. None of the coordinate vertices (that is, 0 dimensional strata of $B$; not to be confused with vertices on the graph) lies on the strict transform $Y$ of $X_{3}$.

We stratify $B=B^{(1)} \supset B^{(2)} \supset \ldots \supset B^{(5)}$. The successive compliments are disjoint unions of tori $\left(T^{r}:=\mathbb{G}_{m}^{r}\right)$

$$
B^{(i)}-B^{(i+1)}=\coprod T^{5-i}
$$

Let us compute some of the motives $T^{r}-Y \cap T^{r}$. For $r=4$, there are two kinds, with $Y \cap T^{4}$ either a graph hypersurface as in fig. 6 or fig. 1 (light edges). In the first case, the graph is a banana graph with 3 edges, subdivided twice. Applying Lemma 8.7 twice, we conclude that $Y \cap T^{4}$ has linear cohomology in the sense of Definition 8.5. The other case is dual to fig. 6 as illustrated in fig. 1, and we may apply Proposition 8.3. The varieties $T^{r}-Y \cap T^{r}$ for $r \leq 3$ are easy to handle. They correspond to shrinking two edges on $\Gamma_{3}$ which yields a graph with 2 vertices, necessarily a banana with tadpoles, or cutting one edge and shrinking another, which yields a "double bubble" (fig. $9)$ with graph polynomial of the form $\left(A_{1}+A_{2}\right)\left(A_{3}+A_{4}\right)$.

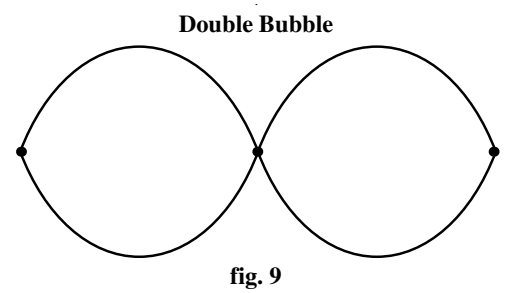

Higher codimensional faces are products of motives associated to graphs with $\leq 2$ vertices. We conclude

Proposition 9.3. Let $(P-Y, B-Y \cap B) \rightarrow\left(\mathbb{P}^{5}-X_{3}, \Delta-\Delta \cap X_{3}\right)$ be obtained by blowing up linear spaces associated to minimal subgraphs of $\Gamma_{3}$ as outlined above. Then all the faces (in all dimensions) of $B-Y \cap B$ have linear cohomology in the sense of Definition 8.5.

\section{RenORMALIZATION}

Consider the "dunce's cap" graph $\Gamma$ (fig. 10). 


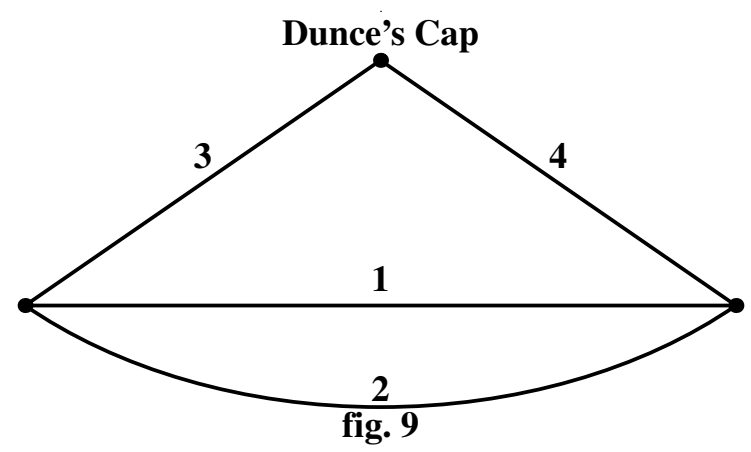

It has 4 edges and 2 loops and hence is log divergent. The reader is encouraged to write down the spanning trees and use Proposition 4.5 to calculate the graph polynomial

$$
\Psi_{\Gamma}=\left(A_{1}+A_{2}\right)\left(A_{3}+A_{4}\right)+A_{1} A_{2}
$$

Among the linear spaces in $X_{\Gamma}$ corresponding to minimal subgraphs which will have to be blown up to define our period is $\left\{A_{1}=A_{2}=0\right\}$. But note the subgraph $e_{1} \cup e_{2}$ is itself log divergent. This means that our form $\omega_{\Gamma}=\Omega / \Psi_{\Gamma}^{2}$ acquires a pole along the exceptional divisor. Indeed, in affine coordinates $a_{i}=A_{i} / A_{4}$ we can write

$$
\omega_{\Gamma}=\frac{d a_{1} d a_{2} d a_{3}}{\left(\left(a_{1}+a_{2}\right)\left(a_{3}+1\right)+a_{1} a_{2}\right)^{2}}
$$

Blowing up introduces affine coordinates $a_{1}, b_{2}=a_{2} / a_{1}, a_{3}$, and we find a log pole along the exceptional divisor which is defined in affine coordinates by $a_{1}=0$.

$$
\omega_{\Gamma}=\frac{d a_{1}}{a_{1}} \wedge \frac{d b_{2} d a_{3}}{\left(\left(1+b_{2}\right)\left(a_{3}+1\right)+a_{1} b_{2}\right)^{2}}
$$

Proposition 10.1 ([BEK],Lemma 5.1). Let $\Gamma$ be a log divergent graph with $m$ edges, and let $\omega_{\Gamma}$ be the associated de Rham form (5.10). Let $\rho: P \rightarrow \mathbb{P}^{m-1}$ be the iterated blowup of linear spaces associated to minimal subgraphs (Examples 7.7(ii)). Then $\Gamma$ has a divergent subgraph if and only if $\rho^{*} \omega_{\Gamma}$ has a pole along some component of the exceptional locus.

When such a pole occurs, the Feynman amplitude (5.10) diverges. Certainly a major theme in physics over the past 50 years has been the program of renormalization to eliminate these poles. Of course one cannot just "eliminate" poles. The problem is rather to isolate in some canonical way the polar terms so that in some ultimate calculation involving a sum over various graphs, the polar terms will all cancel. The theory of limiting mixed Hodge structures offers a possible solution 
in the case that the pole orders along exceptional divisors are all $\leq 1$. (Precisely which pole orders occur depend on which graphs occur in the theory, and this depends on the Lagrangian.)

Suppose $\Gamma$ is a log divergent graph with $2 n$ edges and $n$ loops. The idea is to define linear forms $\ell_{i, t}, 1 \leq i \leq 2 n$ in $A_{1}, \ldots, A_{2 n}$ with coefficients in $\mathbb{R}[t]$ such that $\ell_{i, 0}=A_{i}$. We then define $\Delta_{t}: \prod_{1}^{2 n} \ell_{i, t}=0$ and the real chain $\sigma_{t}: \ell_{i, t} \geq 0$ for $0 \leq t<<1$. We can do this is such a way that $\sigma_{t}$ is contained in the locus where all coordinates are $>0$ for $0<t<<1$. In particular, $\sigma_{t} \cap X_{\Gamma}=\emptyset$ for $0<t<<1$ so $\int_{\sigma_{t}} \omega_{\Gamma}$ converges for $t>0$. What the theory of limiting Hodge structures suggests is that one can find relative cycles $\sigma_{1, t}=\sigma_{t}, \sigma_{2, t}, \ldots, \sigma_{p, t}$ representing classes in $H_{2 n-1}\left(\mathbb{P}^{2 n-1}-X_{\Gamma}, \Delta_{t}-\Delta_{t} \cap X_{\Gamma}, \mathbb{Q}\right)$ and a strictly upper triangular $p \times p$ matrix $N$ with constant coefficients such that (writing superscript $T$ for transpose) the limit

$$
\lim _{t \rightarrow 0} \exp (N \log t)\left(\int_{\sigma_{1, t}} \omega_{\Gamma}, \ldots, \int_{\sigma_{p, t}} \omega_{\Gamma}\right)^{T}
$$

exists.

In broad strokes, the program would be the following. Write $D=$ $\{|t|<\epsilon<<1\}$ for some small disk. Think of a family $\mathbb{P}^{2 n-1}-X_{\Gamma}, \Delta_{t}$ over $D$. At $t=0$ one blows up in $\mathbb{P}^{2 n-1} \times D$ linear spaces contained in $X_{\Gamma} \times\{0\}$ according to the pattern we have used. The resulting family $\mathcal{P}$ over $D$ will contain our blowup $P$ of $\mathbb{P}^{2 n-1}$ as one component of the special fibre. Let $E \subset B \subset P$ be the exceptional components associated to divergent subgraphs. We have seen that $E$ can be stratified in such a way that the strata look like products of $\mathbb{P}^{m_{\mu}}-X_{\gamma}$ for suitable subquotient graphs $\gamma$ of $\Gamma$. Inside each of these factors of strata imagine fixing a $\sigma_{t, \gamma}$ which is a deformation of the reference simplex. Let $\mathcal{T} \subset \mathbb{P}^{2 n-1} \times D^{\times}$be a (punctured) tubular neighborhood of the special fibre $\mathcal{P}_{0}$. $\mathcal{T}$ contracts onto $\mathcal{P}_{0}$ and we write $\pi: \partial \mathcal{T} \rightarrow \mathcal{P}_{0}$ for the restriction to the boundary of $\mathcal{T}$. By definition, the tube over a chain $c$ on $\mathcal{P}_{0}$ is $\pi^{-1}(c) \subset \partial \mathcal{T}$. Given a chain on a stratum of $E$, we can do a similar tube construction to get a chain on a larger stratum. By iterating this game, our products of $\sigma_{t, \gamma}$ get promoted to $2 n-1$-chains supported in $\partial \mathcal{T}$. It seems that this can be done in such a way that the boundary of these chains lies in $\Delta_{t} \cap \partial \mathcal{T}$. (I have only checked this carefully in the special case where $\Delta$ can be deformed while leaving fixed the linear spaces associated to divergent subgraphs, but I conjecture it is true generally.) The resulting chains are the $\sigma_{i, t}$.

In the (very) special case of the dunce's cap (fig. 10) the only divergent subgraph is the circle graph $\gamma=e_{1} \cup e_{2}$, so $E \cong \mathbb{P}^{1} \times \mathbb{P}^{1}$ above has one component given by the blowup of the line $A_{1}=A_{2}=0$ in $\mathbb{P}^{3}$. 
The quotient $\Gamma / \gamma=e_{3} \cup e_{4}$ is also a circle with 2 edges. The Feynman periods for these circle graphs converge

$$
\int_{0}^{\infty} \frac{d\left(A_{1} / A_{2}\right)}{\left(A_{1} / A_{2}+1\right)^{2}}=\int_{0}^{\infty} \frac{d\left(A_{3} / A_{4}\right)}{\left(A_{3} / A_{4}+1\right)^{2}}=1,
$$

so the chains $\sigma_{\gamma}$ and $\sigma_{\Gamma / \gamma}$ can be taken just to be $[0, \infty]$ independent of $t$. The tube $\pi^{-1}([0, \infty] \times[0, \infty])$ can be taken to have boundary lying on the strict transform of $\Delta: A_{1} A_{2} A_{3} A_{4}=0$. Finally, by standard differential topology, $\partial \mathcal{T}$ can be taken to be transversal to the faces of $\Delta$ so a small modification $\Delta_{t}$ of $\Delta$ is still transversal, and the relative homology class of the tube is canonically defined. Call the resulting chain $\sigma_{2, t}$ and let $\sigma_{1, t}$ be the deformation of $\sigma$ as above. For example if I take $\Delta_{t}:\left(A_{1}-t A_{4}\right)\left(A_{2}-t^{2} A_{4}\right) A_{3} A_{4}=0 \mathrm{I}$ find

$$
\begin{aligned}
\int_{\sigma_{1, t}} \omega_{\Gamma} & =\int_{t}^{\infty} \frac{d a_{1}}{a_{1}} \int_{[t, \infty] \times[0, \infty]} \frac{d b_{2} d a_{3}}{\left(\left(1+b_{2}\right)\left(1+a_{3}\right)+a_{1} b_{2}\right)^{2}} \\
\int_{\sigma_{2, t}} \omega_{\Gamma} & =\int_{\left|a_{1}\right|=\epsilon} \frac{d a_{1}}{a_{1}} \int_{[t, \infty] \times[0, \infty]} \frac{d b_{2} d a_{3}}{\left(\left(1+b_{2}\right)\left(1+a_{3}\right)+a_{1} b_{2}\right)^{2}}
\end{aligned}
$$

Define $N=\left(\begin{array}{cc}0 & \frac{1}{2 \pi i} \\ 0 & 0\end{array}\right)$. Then

$$
\lim _{t \rightarrow 0} \exp (N \log t)\left(\begin{array}{c}
\int_{\sigma_{1, t}} \omega_{\Gamma} \\
\int_{\sigma_{2, t}} \omega_{\Gamma}
\end{array}\right)=\left(\begin{array}{c}
\lim _{t \rightarrow 0}\left(\int_{\sigma_{1, t}}+\log t\right) \\
2 \pi i
\end{array}\right)
$$

and this limit exists.

Quite generally, it seems to be possible to give such an ad hoc renormalization scheme when $\Gamma$ is $\log$ divergent with at worst log divergent subgraphs. I suspect this is more or less equivalent to techniques already known to the physicists. The interesting question is whether this is really a limiting Hodge structure. (I am endebted to Hélène Esnault for pointing out that this is a problem.) For this to be the case, we need that the cohomology class $\left[\Omega / \Psi_{\Gamma}^{2}\right]$ should satisfy a certain nilpotent residue condition $[\mathrm{D}]$. By a criterion of Katz, this residue condition will hold if the form $\Omega / \Psi_{\Gamma}^{2}$ has at worst log poles when we blow up on $\mathcal{P}$ on $\mathcal{P}_{0}$ to get a normal crossings situation. Unfortunately, it is very difficult to control these blowups because the singularities of $X_{\Gamma}$ are highly non-linear. However, there is a theory of Hodge modules due to M. Saito [Sa1], [Sa2] which has the following consequence. Change notation so $\mathcal{P}$ becomes a normal crossings model of our situation. Let $\psi$ (not to be confused with $\Psi_{\Gamma}$ ) be the vanishing cycle sheaf on $\mathcal{P}_{0}$. By definition, $\psi$ is an object in the derived category of constructible sheaves on $\mathcal{P}_{0}$ and its cohomology carries a mixed Hodge structure 
(depending on the choice of parameter $t$ ) which is the limiting Hodge structure. As a consequence of the existence of pullbacks for Hodge modules, it will be the case that for any Zariski open set $U_{0} \subset \mathcal{P}_{0}$ that $H^{*}\left(U_{0},\left.\psi\right|_{U_{0}}\right)$ will also carry a Hodge structure. It is known from work of Steenbrink [I] 2.2.4.1 that $\psi \otimes \mathbb{C}$ is quasi-isomorphic to the complex of relative $\log$ forms, the quasi-isomorphism depending on the choice of a parameter $t$. But on $U_{0}$ our form $\Omega / \Psi_{\Gamma}^{2}$ defines a section of top degree in this complex, and hence a class in $H^{2 n-1}\left(U_{0}, \psi \otimes \mathbb{C}\right)$. The challenge would be to show that a period associated with this class coincides with the limit as $t \rightarrow 0$ of the top entry in the column vector (10.4).

Needless to say, the above is more of a statement of a program rather than an outline of results obtained.

\section{Graphs and Zeta Values}

Here are a couple of examples taken from a lecture of Kreimer $[\mathrm{K}]$
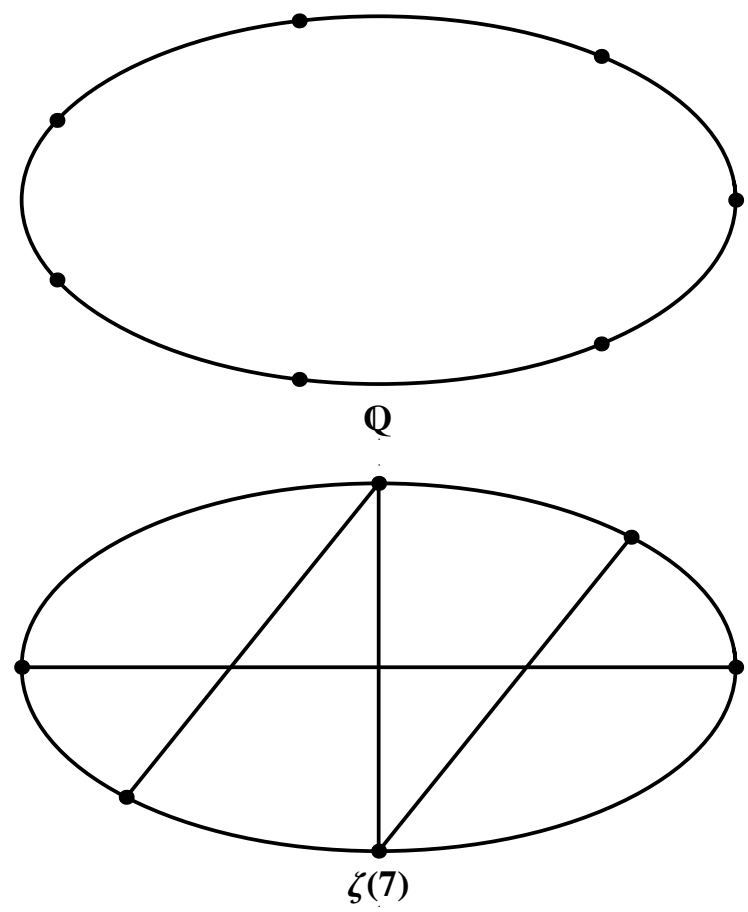


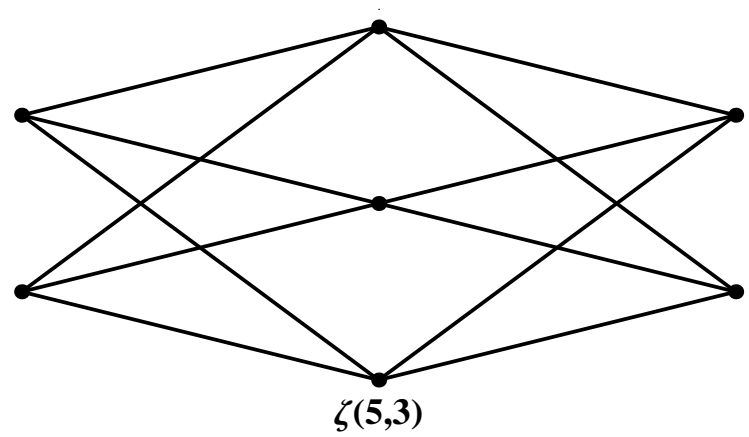

REFERENCES

[BB] Belkale,P., Brosnan,P.: Matroids, Motives, and a Conjecture of Kontsevich, Duke Math. J. 116 (2003), no. 1, 147-188.

[D] Deligne, P.: Local Behavior of Hodge Structures at Infinity (preprint).

[BK1] Broadhurst, D., Kreimer,D.: Knots and numbers in $\Phi^{4}$ theory to 7 loops and beyond, Int. J. Mod. phys. C 6, 519 (1995).

[BK2] Association of multiple zeta values with positive knots via Feynman diagrams upto 9 loops, Phys. Lett. B 393 (3-4) (1997), 403-412.

[P] Polyak, M; Feynman Diagrams for Pedestrians and Mathematicians, arXiv:math.GT/0406251.

[IZ] Itzykson, C., Zuber,J.-B.: Quantum Field Theory, McGraw-Hill (1980).

[BEK] Bloch, S., Esnault, H., Kreimer, D.: Motives associated to Graph Polynomials, to appear in Comm. Math. Physics.

[I] Illusie, L.: Autour du théorème de monodromie locale, in Astérisque 223 (1994).

[Sa1] Saito, M.: Modules de Hodge polarisables, Publ. RIMS, Kyoto Univ. 24, (1988), 849-995.

[Sa2] Saito, M.: Mixed Hodge modules, Publ. RIMS, Kyoto Univ. 26, (1990), 221333.

[St1] Steenbrink, J.: Limits of Hodge Structures, Inv. Math. 31, (1976), 229-257.

[SZ] Steenbrink, J, Zucker, S.:Variation of Mixed Hodge Structure I, Inv. Math. 80, (1985), 489-542.

[GM] Goncharov, A., Manin,Y.: Multiple zeta motives and moduli spaces $\bar{M}_{0, n}$, Comp. Math. 140 (2004), no. 1, 1-14.

[Stem] Stembridge, J.: Counting Points on Varieties over Finite Fields Related to a Conjecture of Kontsevich, Ann. Comb. 2 (1998), 365-385.

[ESV] Esnault, H.,Schechtman, V., Viehweg, E.: Cohomology of local systems on the complement of hyperplanes, Inv. math. 109 (1992), 557-561. Erratum: Inv. math. 112 (1993), 447.

[So] Soulé, C.: Régulateurs, Sem. Bourbaki, Vol. 1984-85. Astérisque 133-134 (1986), 237-253.

[Br] Brown, F.: Multiple zeta values and periods of moduli spaces $\bar{M}_{0, n}(\mathbb{R})$, thèse, (to appear).

[CM] Connes, A., Marcolli, M.: Quantum Fields and Motives, J. Geom. Phys.5 $6(2006)$, no. $1,55-85$. 
[K] Kreimer, D.: Lecture at IHES (2005).

[CK1] Connes, A., Kreimer, D.:Renormalization in Quantum Field Theory and the Riemann-Hilbert Problem I, The Hopf Algebra structure of graphs and the main theorem, Comm. Math. Phys. 210 (2000), no. 1, 249-273.

[CK2] Connes, A., Kreimer, D.: Renormalization in Quantum Field Theory and the Riemann-Hilbert Problem II, The $\beta$ function, diffeomorphisms and the renormalization group Comm. Math. Phys. 216 (2001), no. 1, 215-241.

[Ko1] Kontsevich, M.: Feynman diagrams and low-dimensional topology, First European Congress of Mathematics, Vol. II (Paris 1992), 97-121 Prog. in Math. 120, Birkhäuser, Basel (1994).

[Ko2] Kontsevich, M.: Formal (non)commutative symplectic geometry, in the Gelfand mathematical Seminars 1990-1992, 173-187, Birkhäuser, Boston (1993).

[CV] Conant,J., Vogtmann, K.: On a Theorem of Kontsevich, Alg. Geo. Topology, 3(2003), 1167-1224.

[OT] Orlik, P., Terao, H.: Arrangements of Hyperplanes, Grundlehren der Math. Wissenschaften. 300, Springer Verlag (1992).

Dept. of Mathematics, University of Chicago, Chicago, IL 60637, USA

E-mail address: bloch@math.uchicago.edu 